\title{
EVALUATION OF ARG-1 SAMPLES PREPARED BY CESIUM CARBONATE DISSOLUTION DURING THE ISOLOK SME ACCEPTABILITY TESTING
}

\author{
C.J. Coleman \\ K.R. Hera \\ T.B. Edwards
}

November 2011

Applied Computational Engineering and Statistics Savannah River National Laboratory

Aiken, SC 29808

This document was prepared in conjunction with work accomplished under Contract No. DE-AC09-08SR22470 with the U.S. Department of Energy.

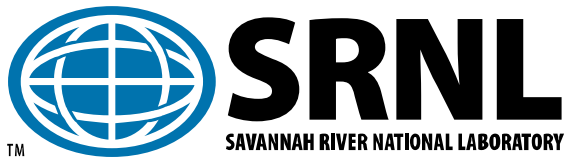




\section{DISCLAIMER}

This work was prepared under an agreement with and funded by the U.S. Government. Neither the U.S. Government or its employees, nor any of its contractors, subcontractors or their employees, makes any express or implied: 1 . warranty or assumes any legal liability for the accuracy, completeness, or for the use or results of such use of any information, product, or process disclosed; or 2. representation that such use or results of such use would not infringe privately owned rights; or 3. endorsement or recommendation of any specifically identified commercial product, process, or service. Any views and opinions of authors expressed in this work do not necessarily state or reflect those of the United States Government, or its contractors, or subcontractors.

This document was prepared in conjunction with work accomplished under Contract No. DE-AC09-08SR22470 with the U.S. Department of Energy. 


\section{REVIEWS AND APPROVALS}

\section{AUTHORS:}

C.J. Coleman, SRNL, Spectroscopy and Separations

Date

Savannah River National Laboratory

K.R. Hera, SRNL, Mechanical Systems \& Custom Equipment Dev.

Date

Savannah River National Laboratory

T.B. Edwards, SRNL, Applied Computational Engineering and Statistics Date

Savannah River National Laboratory

\section{TECHNICAL REVIEWER:}

R.L. Walker, SRNL, Applied Computational Engineering and Statistics Date Savannah River National Laboratory

\section{APPROVERS}

P. L. Lee, Manager, Applied Computational Engineering and Statistics

Date Savannah River National Laboratory

C. C. Herman, Manager, Process Technology Programs

Date

Savannah River National Laboratory

R. T. McNew, Manager, Waste Laboratory Services

Date

Savannah River Remediation

J. E. Occhipinti, Manager, Waste Solidification Engineering

Date

Savannah River Remediation 
SRNL-STI-2011-00711

Revision 0

\section{EXECUTIVE SUMMARY}

Evaluation of the Defense Waste Processing Facility (DWPF) Chemical Process Cell (CPC) cycle time identified several opportunities to improve the CPC processing time. The Mechanical Systems \& Custom Equipment Development (MS\&CED) Section of the Savannah River National Laboratory (SRNL) recently completed the evaluation of one of these opportunities - the possibility of using an Isolok sampling valve as an alternative to the Hydragard valve for taking DWPF process samples at the Slurry Mix Evaporator (SME). The use of an Isolok for SME sampling has the potential to improve operability, reduce maintenance time, and decrease CPC cycle time. The SME acceptability testing for the Isolok was requested in Task Technical Request (TTR) HLW-DWPF-TTR-2010-0036 and was conducted as outlined in Task Technical and Quality Assurance Plan (TTQAP) SRNL-RP-2011-00145. RW-0333P QA requirements applied to the task, and the results from the investigation were documented in SRNL-STI-2011-00693.

The objective of that study was to qualify the Isolok for use in sampling the Slurry Mix Evaporator (SME) tank at the DWPF. Measurement of the chemical composition of study samples was a critical component of the SME acceptability testing of the Isolok. A sampling and analytical plan, SRNL-RP-2011-00294, supported the investigation with the analytical plan directing that the study samples be prepared by a cesium carbonate $\left(\mathrm{Cs}_{2} \mathrm{CO}_{3}\right)$ fusion dissolution method and analyzed by Inductively Coupled Plasma - Optical Emission Spectroscopy (ICP-OES). The use of the cesium carbonate preparation method for the Isolok testing provided an opportunity for an additional assessment of this dissolution method, which is being investigated as a potential replacement for the two methods (i.e., sodium peroxide fusion and mixed acid dissolution) that have been used at the DWPF for the analysis of SME samples. The $\mathrm{Cs}_{2} \mathrm{CO}_{3}$ testing associated with the Isolok testing does provide additional insight into the performance of the method as conducted by SRNL. The performance is to be investigated by looking to the composition measurement data generated by the samples of a standard glass, the Analytical Reference Glass - 1 (ARG-1), that were prepared by the $\mathrm{Cs}_{2} \mathrm{CO}_{3}$ method and included in the SME acceptability testing of the Isolok. The measurements of theses samples were presented in SRNL-STI2011-00693, but no statistical analysis of these measurements was conducted as part of those results.

The ARG-1 measurements, as weight percent (wt\%) oxides, generated during the SME acceptability testing for the Isolok are statistically analyzed in this report. Three sources of variation in these measurements were explored: variation due to preparation block effects, variation due to ICP-OES analytical block effects, and within block variation for these measurements, where the within block variation is due to the repeatability of the preparation process for a given preparation block and to the variation in the measurements during an analytical block of work that is due to the repeatability of the ICP-OES measurement process. These results suggest that differences among the preparation blocks played an insignificant role in the variation seen in the ARG-1 results over the course of the Isolok testing. From this investigation, the components of variation (i.e., the analytical block-to-block effects and the within-block effects) as \% relative standard deviations are less than $5 \%$ for those oxides at concentrations of $0.1 \mathrm{wt} \%$ or greater. A bound on the bias in the $\mathrm{Cs}_{2} \mathrm{CO}_{3}$ method of its measurements for each oxide of interest value is also provided at a 95\% confidence. For those oxides present in ARG-1 at concentrations greater than or equal to $0.1 \mathrm{wt} \%$, only $\mathrm{B}_{2} \mathrm{O}_{3}$ has a bias bound (its value is $9.84 \%$ ) that is larger than $4.41 \%$ (the value for $\mathrm{MnO}$ ). Thus, only $\mathrm{B}_{2} \mathrm{O}_{3}$ has a bias bound that is larger than $5 \%$.

The low bias for boron measurements is almost certainly due to volatilization of boron at the $1050{ }^{\circ} \mathrm{C}$ fusion conditions of the $\mathrm{Cs}_{2} \mathrm{CO}_{3}$ method. Cesium species also can be volatilized under these conditions and it is believed that a carrier distillation effect results in the loss of boron. The relatively high fusion temperature was used in anticipation of needing very rigorous fusion conditions for dissolving a 1-1.5 gram wafer of glass that potentially results from vitrifying a $3 \mathrm{~mL}$ SME sample taken with the Isolok sampler. Experiments performed at the DWPF Laboratory and at SRNL indicate that the low boron bias from the $\mathrm{Cs}_{2} \mathrm{CO}_{3}$ method is essentially eliminated when the method is carried out at $900{ }^{\circ} \mathrm{C}$. The lower fusion temperature is effective for attacking powdered glass and small shards of glass, but may not result in complete attack of a1.5 gram wafer of glass. 


\section{TABLE OF CONTENTS}

LIST OF ABBREVIATIONS .............................................................................. vi

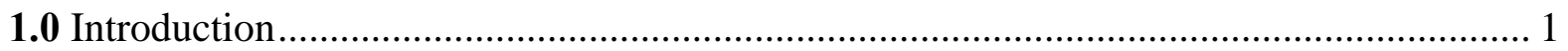

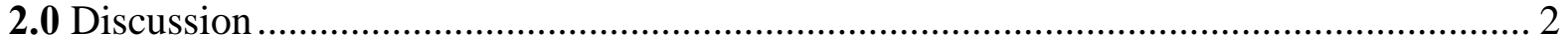

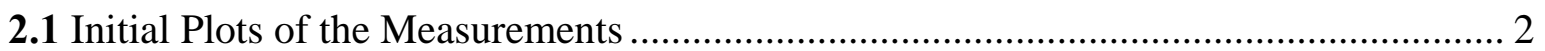

2.2 Preparation Block versus ICP-OES Analytical Block Variation................................... 2

2.3 ICP-OES Analytical Block Variation..................................................................... 4

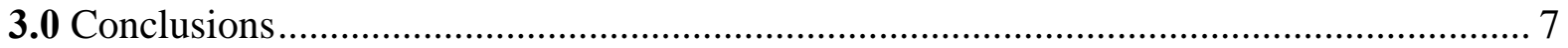

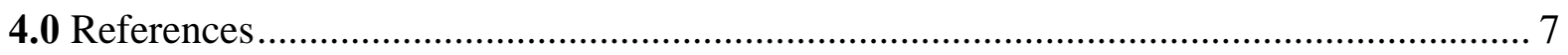

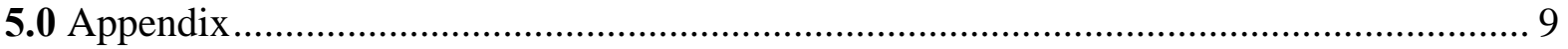




\section{LIST OF ABBREVIATIONS}

$\begin{array}{ll}\text { ARG-1 } & \text { Analytical Reference Glass - 1 } \\ \text { DWPF } & \text { Defense Waste Processing Facility } \\ \text { ICP-OES } & \text { Inductively Coupled Plasma - Optical Emission Spectroscopy } \\ \text { JMP } & \text { Statistical software package from SAS Institute, Inc. } \\ \text { SME } & \text { Slurry Mix Evaporator } \\ \text { SRNL } & \text { Savannah River National Laboratory } \\ \text { SRR } & \text { Savannah River Remediation, LLC } \\ \text { TTR } & \text { Technical Task Request } \\ \text { TTQAP } & \text { Task Technical and Quality Assurance } \\ \text { wt\% } & \text { Weight Percent }\end{array}$


SRNL-STI-2011-00711

Revision 0

\subsection{Introduction}

Evaluation of Defense Waste Processing Facility (DWPF) Chemical Process Cell (CPC) cycle time identified several opportunities to improve the CPC processing time. The Mechanical Systems \& Custom Equipment Development (MS\&CED) Section of the Savannah River National Laboratory (SRNL) recently completed the evaluation of one of these opportunities - the possibility of using an Isolok sampling valve as an alternative to the Hydragard valve for taking DWPF process samples at the Slurry Mix Evaporator (SME). The use of an Isolok for SME sampling has the potential to improve operability, reduce maintenance time, and decrease CPC cycle time. The SME acceptability testing for the Isolok was requested in Task Technical Request (TTR) HLW-DWPF-TTR-2010-0036 [1] and was conducted as outlined in Task Technical and Quality Assurance Plan (TTQAP) SRNLRP-2011-00145 [2]. RW-0333P QA requirements applied to the task, and the results from the investigation were documented in SRNL-STI-2011-00693 [3].

Measurement of the chemical composition of study samples was a critical component of the SME acceptability testing of the Isolok. A sampling and analytical plan [4] supported the investigation with the analytical plan directing that the study samples be prepared by a cesium carbonate $\left(\mathrm{Cs}_{2} \mathrm{CO}_{3}\right)$ fusion dissolution method and analyzed by Inductively Coupled Plasma - Optical Emission Spectroscopy (ICP-OES). The use of the cesium carbonate preparation method for the Isolok testing provided an opportunity for an additional assessment of this dissolution method, which is being investigated as a potential replacement for the two methods (i.e., sodium peroxide fusion and mixed acid dissolution) that have been used at the DWPF for the analysis of SME samples. Earlier testing of the $\mathrm{Cs}_{2} \mathrm{CO}_{3}$ method yielded promising results [5]-[7] which led to a TTR [8] from Savannah River Remediation, LLC (SRR) to SRNL for additional support and an associated TTQAP [9] to direct the SRNL efforts. A technical report [8] resulting from this work was issued that recommended that the mixed acid method be replaced by the $\mathrm{Cs}_{2} \mathrm{CO}_{3}$ method for the measurement of magnesium $(\mathrm{Mg})$, sodium $(\mathrm{Na})$, and zirconium $(\mathrm{Zr})$ with additional testing of the method by DWPF Laboratory being needed before further implementation of the $\mathrm{Cs}_{2} \mathrm{CO}_{3}$ method at that laboratory.

While the SME acceptability testing of the Isolok does not address any of the open issues remaining after the publication of the recommendation for the replacement of the mixed acid method by the $\mathrm{Cs}_{2} \mathrm{CO}_{3}$ method [10] (since those issues are to be addressed by the DWPF Laboratory), the $\mathrm{Cs}_{2} \mathrm{CO}_{3}$ testing associated with the Isolok testing does provide additional insight into the performance of the method as conducted by SRNL. The performance is to be investigated by looking to the composition measurement data generated by the samples of a standard glass, the Analytical Reference Glass - 1 (ARG-1), that were prepared by the $\mathrm{Cs}_{2} \mathrm{CO}_{3}$ method and included in the SME acceptability testing of the Isolok. The measurements of these samples were presented as part of the study results [3], but no statistical analysis of these measurements was conducted as part of those results. It is the purpose of this report to provide that analysis, which was supported using JMP Version 7.0.2 [11]. 


\subsection{Discussion}

The ARG-1 measurements, as weight percent (wt\%) oxides, that are of interest in this investigation are provided in Table A1 in the Appendix. The values were generated by the analytical plan [4] that was issued to support the SME acceptability testing conducted for the Isolok. Table A2 in the Appendix provides the reference composition of the ARG-1 standard as wt\% oxides.

\subsection{Initial Plots of the Measurements}

Exhibit A1 in the Appendix provides a series of plots by oxide of the measurements of Table A1 grouped by test phase, preparation groupings, and analytical groupings. The sample ID and the targeted value for each oxide from Table A2 are also shown as part of the information on the x-axis of each plot. The average of all of the measurements over all three test phases is shown on each plot as a horizontal line. Included in this exhibit is a plot of the sums of oxides for the measurements.

Some interesting observations can be made for the sum of oxides plot. As seen in this plot, the targeted sum for the ARG-1 oxides of interest in this report was $99.43 \mathrm{wt} \%$. Eight of the 54 sums (i.e., 14.8\%) fall outside of the interval from 95 to $105 \mathrm{wt} \%$, where this interval is typically used as one of the metrics in assessing the analytical process that was used to generate the chemical composition measurements. There are two aspects of these results that are worth noting here. One is that those samples showing a low sum of oxides also show low recoveries for many of the other oxides, not just $\mathrm{SiO}_{2}$. Of even more interest is the fact that 6 of the 8 samples that have a sum that falls below the 95\% value are actually measured in two analytical blocks but their sum only falls below the 95\% value in one of the two blocks. See Table 1. This suggests that the preparation of the samples was not an issue in the low sums of oxides seen for some of the results, but the likely problem was the performance of the ICP-OES instrumentation or human errors such mislabeling of sample bottles or an error during sample dilution. There is also a suggestion that one of the bigger contributors to the variation seen in the measurements for all of the oxides is the repeatability of the measurements by the ICP-OES instrumentation. The sources of variation are investigated in the next section.

Table 1. A Subset of the Sums of Oxides Values

\begin{tabular}{|ccccc|}
\hline Test & Preparation Block & Analytical Block & Sample ID & Measurement \\
\hline Phase 1 & 2 & 2 & ARG-1B22 & 99.62 \\
Phase 1 & 2 & 5 & ARG-1B22 & 91.63 \\
\hline Phase 1 & 3 & 3 & ARG-1B33 & 102.38 \\
Phase 1 & 3 & 6 & ARG-1B33 & 94.27 \\
\hline Phase 2 & 2 & 2 & ARG-1B21 & 92.86 \\
Phase 2 & 2 & 5 & ARG-1B21 & 98.08 \\
\hline Phase 2 & 2 & 2 & ARG-1B23 & 98.93 \\
Phase 2 & 2 & 5 & ARG-1B23 & 93.79 \\
\hline Phase 2 & 3 & 3 & ARG-1B31 & 93.92 \\
Phase 2 & 3 & 6 & ARG-1B31 & 98.00 \\
\hline Phase 2 & 3 & 3 & ARG-1B33 & 101.05 \\
Phase 2 & 3 & 6 & ARG-1B33 & 92.75 \\
\hline
\end{tabular}

\subsection{Preparation Block versus ICP-OES Analytical Block Variation}

For those ARG-1 samples that were measured twice during the Isolok testing, once in each of two different ICP-OES analytical blocks, there is an opportunity to estimate the variation in these measurements due to preparation block effects and the variation due the ICP-OES analytical block effects. These variations are assessed relative to the within block variation for these measurements, 
where the within block variation is due to the repeatability of the preparation process for a given preparation block and to the variation in the measurements during an analytical block of work that is due to the repeatability of the ICP-OES measurement process. The statistical model for the measurement of each of the oxides of interest that facilitates the estimation of the components of variance is given by:

Equation 1.

$$
\mathrm{y}_{\mathrm{ijk}}=\mu+\mathrm{p}_{\mathrm{i}}+\mathrm{a}_{\mathrm{j}(\mathrm{i})}+\mathrm{e}_{\mathrm{ijk}}
$$

where

$\mathrm{y}_{\mathrm{ijk}} \quad$ is the measurement of the $\mathrm{k}^{\text {th }}$ sample in analytical block $\mathrm{j}$ for a sample prepared in preparation block i,

$\mu \quad$ represents the average measurement for the given oxide,

$\mathrm{p}_{\mathrm{i}} \quad$ represents a random effect for the $\mathrm{i}^{\text {th }}$ preparation block that captures block-to-block effects,

$\mathrm{a}_{\mathrm{j}(\mathrm{i})} \quad$ represents a random effect for the $\mathrm{j}^{\text {th }}$ analytical block nested within the $\mathrm{i}^{\text {th }}$ preparation block, this random effect captures block-to-block effects for the analytical process, and

$\mathrm{e}_{\mathrm{ijk}} \quad$ represents the within block variation, and as described above this variation is due to the repeatability of the preparation process for a given preparation block and to the variation in the measurements during an analytical block of work that is due to the repeatability of the ICP-OES measurement process.

The a's in this model are assumed to be normally distributed with a zero mean and with a constant but unknown standard deviation that may be represented by $\sigma_{\mathrm{a}}$. The p's in the model are assumed to be normally distributed with a zero mean and with a constant but unknown standard deviation that may be represented by $\sigma_{\mathrm{p}}$. The e's in this model are random effects that are assumed to be normally distributed with a zero mean and with a constant but unknown standard deviation that may be represented by $\sigma_{\mathrm{e}}$.

To facilitate the analysis of this model using JMP, the preparation blocks and analytical blocks were uniquely tied to the test phases as indicated in Exhibit 1. The results from the JMP analysis are provided in Exhibit A2 in the Appendix, and they include tests for the statistical significance of two components of variation of the model in equation (1) (i.e., $\sigma_{p}$ and $\sigma_{a}$ ) relative to the size of the within block component, i.e., the standard deviation, $\sigma_{\mathrm{e}}$. The outcome of one of these tests indicates a statistically significant component of variation by its p-value, which is labeled as "Prob $>\mathbf{F}$ " in the portion of the results with the heading "Tests wrt Random Effects." When the p-value is less than 0.05, then the estimated component of variation is statistically significant at the $5 \%$ level. To help with this interpretation, the p-values in Exhibit A2 have been shaded: green if the variance component is not statistically significant and red if it is. The estimated variance for the analytical blocks is statistically significant for several of the oxides (specifically, $\mathrm{B}_{2} \mathrm{O}_{3}, \mathrm{Li}_{2} \mathrm{O}, \mathrm{MgO}, \mathrm{MnO}$, and $\mathrm{NiO}$ ), while the estimated variance for the preparation blocks is statistically significant for only $\mathrm{CuO}$ and $\mathrm{ZnO}$ (two minor oxides for ARG-1). These results suggest that differences among the preparation blocks played an insignificant role in the variation seen in the ARG-1 results over the course of the Isolok testing. This allows for the use of a less complex model with a more complete set of the measurements. 


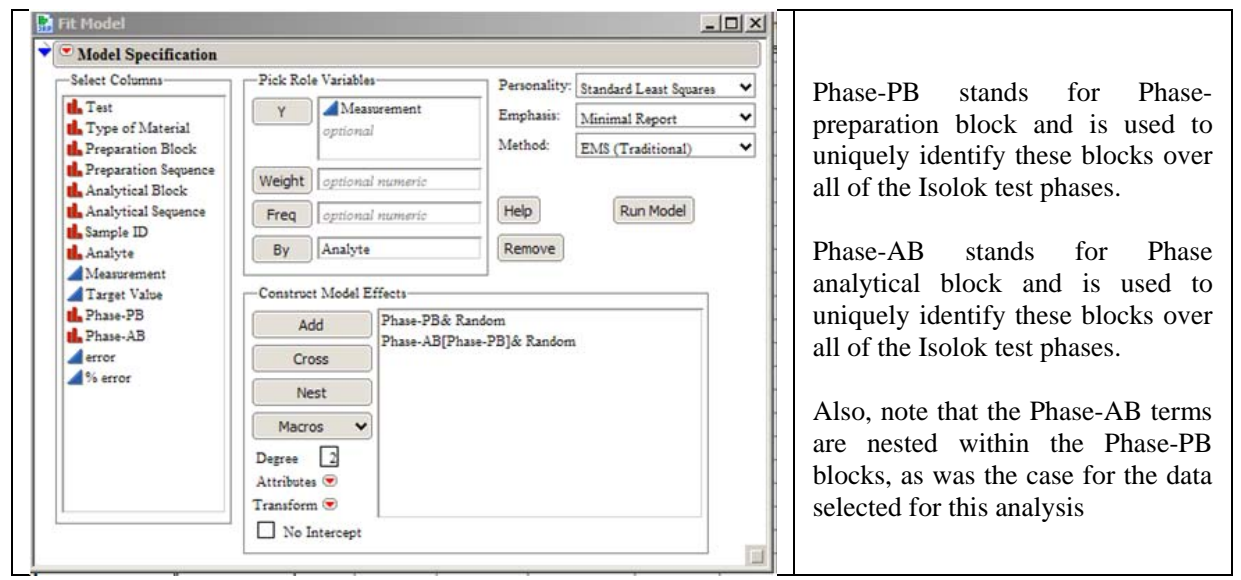

Exhibit 1. Nested, Random Effects Model for Preparation and Analytical Block Effects

\subsection{ICP-OES Analytical Block Variation}

In this section two sources for the variation in the ARG-1 measurements is investigated: an ICP-OES analytical block-to-block effect and the within analytical block variation. For this analysis, all of the ARG-1 measurements were used. Once again, the within block variation is due to the repeatability of the preparation process for a given preparation block and to the variation in the measurements during an analytical block of work that is due to the repeatability of the ICP-OES measurement process. The statistical model for the measurement of each of the oxides of interest that facilitates the estimation of the components of variance is given by:

\section{Equation 1.}

$$
\mathrm{y}_{\mathrm{ij}}=\mu+\mathrm{a}_{\mathrm{i}}+\mathrm{e}_{\mathrm{ij}}
$$

where

$\mathrm{y}_{\mathrm{ij}} \quad$ is the measurement of the $\mathrm{j}^{\mathrm{th}}$ sample in analytical block $\mathrm{i}$ for a prepared sample,

$\mu \quad$ represents the average measurement for the given oxide,

$\mathrm{a}_{\mathrm{i}} \quad$ represents a random effect for the $\mathrm{i}^{\text {th }}$ analytical block that captures block-to-block effects,

$\mathrm{e}_{\mathrm{ij}} \quad$ represents the within block variation, and as described above this variation is due to the repeatability of the preparation process for a given preparation block and to the variation in the measurements during an analytical block of work that is due to the repeatability of the ICP-OES measurement process.

The a's in this model are assumed to be normally distributed with a zero mean and with a constant but unknown standard deviation that may be represented by $\sigma_{a}$. The e's in this model are random effects that are assumed to be normally distributed with a zero mean and with a constant but unknown standard deviation that may be represented by $\sigma_{\mathrm{e}}$.

To facilitate the analysis of this model using JMP, the analytical blocks were uniquely tied to the test phases as indicated in Exhibit 2. The results from the JMP analysis are provided in Exhibit A3 in the Appendix, and they include a test for the statistical significance of the $\sigma_{\mathrm{a}}$ variation of the model in equation (1) relative to the size of the within block variation, i.e., the standard deviation, $\sigma_{\mathrm{e}}$. The outcome of one of these tests indicates a statistically significant component of variation by its p-value, which is labeled as "Prob $>$ F" in the portion of the results with the heading "Tests wrt Random 
Effects." When the p-value is less than 0.05, then the estimated block-to-block component of variation is statistically significant at the 5\% level. To help with this interpretation, the p-values in Exhibit A3 have been shaded: green if the variance component is not statistically significant and red if it is. The estimated variance for the analytical blocks is statistically significant for several of the oxides (especially, $\mathrm{B}_{2} \mathrm{O}_{3}, \mathrm{BaO}, \mathrm{CaO}, \mathrm{Cr}_{2} \mathrm{O}_{3}, \mathrm{CuO}, \mathrm{Li}_{2} \mathrm{O}, \mathrm{MgO}, \mathrm{MnO}$, and $\mathrm{NiO}$ ). These results suggest that differences among the analytical blocks can play a significant role in the variation seen in the ARG-1 results.

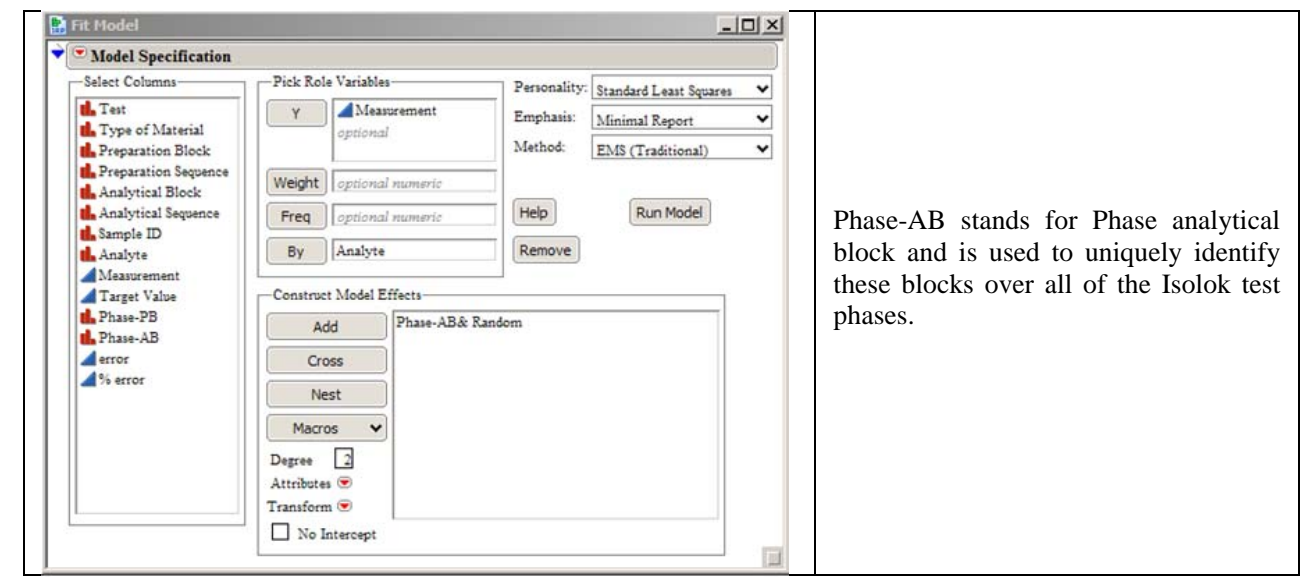

Exhibit 2. Random Effects Model for Analytical Block Effects

In Table 2, the information from Exhibit A3 for each oxide is summarized and used to determine a $95 \%$ confidence interval for the mean of the measurements for each oxide. If the confidence interval for an oxide does not contain the reference concentration for that oxide (also provided in Table 2), then there is a statistically significant bias (at the $5 \%$ level) in the measurements. The results in Table 2 indicate that there are biases for all of the oxides except $\mathrm{CaO}, \mathrm{K}_{2} \mathrm{O}, \mathrm{Na}_{2} \mathrm{O}, \mathrm{SiO}_{2}$, and $\mathrm{TiO}_{2}$. Regardless of the statistical significance of the bias, a bound on its value (at a 95\% confidence) is also provided in Table 2. For those oxides present in ARG-1 at concentrations greater than or equal to 0.1 $\mathrm{wt} \%$, only $\mathrm{B}_{2} \mathrm{O}_{3}$ has a bias bound (its value is $9.84 \%$ ) that is larger than $4.41 \%$ (the value for $\mathrm{MnO}$ ). Thus, only $\mathrm{B}_{2} \mathrm{O}_{3}$ has a bias bound that is larger than $5 \%$.

The low bias for boron measurements is almost certainly due to volatilization of boron at the $1050{ }^{\circ} \mathrm{C}$ fusion conditions of the $\mathrm{Cs}_{2} \mathrm{CO}_{3}$ method. Cesium species also can be volatilized under these conditions, and it is believed that a carrier distillation effect results in the loss of boron. The relatively high fusion temperature was used in anticipation of needing very rigorous fusion conditions for dissolving a 1-1.5 gram wafer of glass that potentially results from vitrifying a $3 \mathrm{~mL}$ SME sample taken with the Isolok sampler. Experiments performed at the DWPF Laboratory and at SRNL indicate that the low boron bias from the $\mathrm{Cs}_{2} \mathrm{CO}_{3}$ method is essentially eliminated when the method is carried out at $900{ }^{\circ} \mathrm{C}$. The lower fusion temperature is effective for attacking powdered glass and small shards of glass, but may not result in complete attack of a1.5 gram wafer of glass.

Also, note that Table 2 presents the components of variation (i.e., the analytical block-to-block effects and the within-block effects) as \% relative standard deviations, and from Table 2, all of these values are less than $5 \%$ for those oxides at concentrations of $0.1 \mathrm{wt} \%$ or greater. 
Table 2. Summary of Results from the Model of Random Analytical Block Effects

\begin{tabular}{|c|c|c|c|c|c|c|c|c|c|c|c|c|c|c|}
\hline \multirow[b]{3}{*}{ Analyte } & \multirow{3}{*}{\begin{tabular}{c|} 
Reference \\
Concentration
\end{tabular}} & \multirow{2}{*}{\multicolumn{2}{|c|}{$\begin{array}{l}\text { Average } \\
\text { Measurec }\end{array}$}} & \multirow{3}{*}{\begin{tabular}{|c|} 
ICP Block \\
to Block \\
Variance \\
\end{tabular}} & \multirow{3}{*}{\begin{tabular}{|l|} 
Residual \\
Variance \\
\end{tabular}} & \multirow{3}{*}{$\begin{array}{c}\text { Total } \\
\text { Variance } \\
\end{array}$} & \multirow{3}{*}{\begin{tabular}{|c|}
$\%$ Relative \\
ICP Block \\
Std. Dev. \\
\end{tabular}} & \multirow{3}{*}{\begin{tabular}{|c|}
$\%$ Relative \\
Residual \\
Std. Dev. \\
\end{tabular}} & \multirow{3}{*}{\begin{tabular}{|c|}
$\%$ Relative \\
Total \\
Std. Dev. \\
\end{tabular}} & \multirow{3}{*}{\begin{tabular}{|c|} 
Mean \\
Square \\
Error \\
\end{tabular}} & \multirow[b]{3}{*}{ DoF } & \multirow{2}{*}{\multicolumn{2}{|c|}{$\begin{array}{c}\text { 95\% Confidence Interval } \\
\text { for the Mean }\end{array}$}} & \multirow{3}{*}{\begin{tabular}{|l|}
$\%$ Bias Bound \\
at $\geq 95 \%$ Conf \\
\end{tabular}} \\
\hline & & & & & & & & & & & & & & \\
\hline & & $\mathbf{n}$ & Value & & & & & & & & & Lower Limit & Upper Limit & \\
\hline $\mathrm{Al}_{2} \mathrm{O}_{3}$ & 4.73 & 54 & 4.607 & $2.854 \mathrm{E}-03$ & $1.400 \mathrm{E}-02$ & $1.685 \mathrm{E}-02$ & $1.2 \%$ & $2.6 \%$ & $2.8 \%$ & 2.26E-02 & 17 & 4.5641 & 4.6504 & $3.51 \%$ \\
\hline $\mathrm{B}_{2} \mathrm{O}_{3}$ & 8.67 & 54 & 7.929 & 2.755E-02 & 6.891E-02 & $9.646 \mathrm{E}-02$ & $2.1 \%$ & $3.3 \%$ & $3.9 \%$ & 1.52E-01 & 17 & 7.8169 & 8.0405 & $9.84 \%$ \\
\hline $\mathrm{BaO}$ & 0.09 & 54 & 0.092 & 3.482E-06 & 1.100E-05 & $1.448 \mathrm{E}-05$ & $2.0 \%$ & $3.6 \%$ & $4.1 \%$ & $2.15 \mathrm{E}-05$ & 17 & 0.0908 & 0.0935 & $3.88 \%$ \\
\hline $\mathrm{CaO}$ & 1.43 & 54 & 1.444 & $4.600 \mathrm{E}-04$ & 1.299E-03 & $1.759 \mathrm{E}-03$ & $1.5 \%$ & $2.5 \%$ & $2.9 \%$ & $2.68 \mathrm{E}-03$ & 17 & 1.4286 & 1.4584 & $1.98 \%$ \\
\hline $\mathrm{Cr}_{2} \mathrm{O}_{3}$ & 0.09 & 54 & 0.098 & 9.885E-06 & 1.425E-05 & $2.414 \mathrm{E}-05$ & $3.2 \%$ & $3.9 \%$ & $5.0 \%$ & 4.39E-05 & 17 & 0.0958 & 0.0996 & $10.66 \%$ \\
\hline $\mathrm{CuO}$ & 0 & 54 & 0.010 & 6.474E-05 & 6.519E-06 & $7.126 \mathrm{E}-05$ & $81.0 \%$ & $25.7 \%$ & $85.0 \%$ & $2.00 \mathrm{E}-04$ & 17 & 0.0059 & 0.0140 & na \\
\hline $\mathrm{Fe}_{2} \mathrm{O}_{3}$ & 14 & 54 & 13.900 & $0.000 \mathrm{E}+00$ & 1.082E-01 & 1.082E-01 & $0.0 \%$ & $2.4 \%$ & $2.4 \%$ & 8.74E-02 & 17 & 13.8153 & 13.9850 & $1.32 \%$ \\
\hline $\mathrm{K}_{2} \mathrm{O}$ & 2.71 & 54 & 2.695 & $0.000 \mathrm{E}+00$ & 1.560E-02 & $1.560 \mathrm{E}-02$ & $0.0 \%$ & $4.6 \%$ & $4.6 \%$ & 1.53E-02 & 17 & 2.6593 & 2.7302 & $1.87 \%$ \\
\hline $\mathrm{Li}_{2} \mathrm{O}$ & 3.21 & 54 & 3.156 & 1.969E-03 & 5.845E-03 & 7.814E-03 & $1.4 \%$ & $2.4 \%$ & $2.8 \%$ & 1.18E-02 & 17 & 3.1249 & 3.1871 & $2.65 \%$ \\
\hline $\mathrm{MgO}$ & 0.86 & 54 & 0.842 & $2.020 \mathrm{E}-04$ & \begin{tabular}{|l|}
$4.260 \mathrm{E}-04$ \\
\end{tabular} & $6.280 \mathrm{E}-04$ & $1.7 \%$ & $2.5 \%$ & $3.0 \%$ & 1.03E-03 & 17 & 0.8324 & 0.8508 & $3.21 \%$ \\
\hline $\mathrm{MnO}$ & 1.89 & 54 & 1.827 & 9.940E-04 & 2.087E-03 & 3.081E-03 & $1.7 \%$ & $2.5 \%$ & $3.0 \%$ & 5.07E-03 & 17 & 1.8066 & 1.8475 & $4.41 \%$ \\
\hline $\mathrm{Na}_{2} \mathrm{O}$ & 11.5 & 54 & 11.445 & 1.559E-02 & 9.152E-02 & $1.071 \mathrm{E}-01$ & $1.1 \%$ & $2.6 \%$ & $2.9 \%$ & 1.38E-01 & 17 & 11.3380 & 11.5515 & $1.41 \%$ \\
\hline $\mathrm{NiO}$ & 1.05 & 54 & 1.018 & 3.310E-04 & \begin{tabular}{|l|}
$6.990 \mathrm{E}-04$ \\
\end{tabular} & $1.030 \mathrm{E}-03$ & $1.8 \%$ & $2.6 \%$ & $3.2 \%$ & 1.69E-03 & 17 & 1.0057 & 1.0293 & $4.22 \%$ \\
\hline $\mathrm{SiO}_{2}$ & 47.9 & 54 & 48.043 & 1.908E-01 & $1.482 \mathrm{E}+00$ & $1.673 \mathrm{E}+00$ & $0.9 \%$ & $2.5 \%$ & $2.7 \%$ & $2.05 \mathrm{E}+00$ & 17 & 47.6316 & 48.4547 & $1.16 \%$ \\
\hline $\mathrm{TiO}_{2}$ & 1.15 & 54 & 1.148 & 7.514E-05 & 8.440E-04 & 9.191E-04 & $0.8 \%$ & $2.5 \%$ & $2.6 \%$ & 1.07E-03 & 17 & 1.1382 & 1.1570 & $1.03 \%$ \\
\hline $\mathrm{ZnO}$ & 0.02 & 54 & 0.024 & 1.115E-08 & \begin{tabular}{|l|}
$1.893 \mathrm{E}-06$ \\
\end{tabular} & 1.904E-06 & $0.4 \%$ & $5.6 \%$ & $5.7 \%$ & 1.93E-06 & 17 & 0.0240 & 0.0248 & $24.04 \%$ \\
\hline $\mathrm{ZrO}_{2}$ & 0.13 & 54 & 0.132 & 2.322E-06 & 2.154E-05 & $2.386 \mathrm{E}-05$ & $1.2 \%$ & $3.5 \%$ & $3.7 \%$ & 2.85E-05 & 17 & 0.1303 & 0.1334 & $2.58 \%$ \\
\hline Sum & 99.43 & 54 & 98.408 & 5.737E-03 & $6.169 \mathrm{E}+00$ & $6.175 \mathrm{E}+00$ & $0.1 \%$ & $2.5 \%$ & $2.5 \%$ & $6.19 \mathrm{E}+00$ & 17 & 97.6938 & 99.1220 & $1.75 \%$ \\
\hline
\end{tabular}




\subsection{Conclusions}

The ARG-1 measurements, as wt\% oxides, generated during the SME acceptability testing for the Isolok are statistically analyzed in this report. Three sources of variation in these measurements were explored: variation due to preparation block effects, variation due the ICP-OES analytical block effects, and within block variation for these measurements, where the within block variation is due to the repeatability of the preparation process for a given preparation block and to the variation in the measurements during an analytical block of work that is due to the repeatability of the ICP-OES measurement process. These results suggest that differences among the preparation blocks played an insignificant role in the variation seen in the ARG-1 results over the course of the Isolok testing. From this investigation, the components of variation (i.e., the analytical block-to-block effects and the within-block effects) as \% relative standard deviations are less than $5 \%$ for those oxides at concentrations of $0.1 \mathrm{wt} \%$ or greater. A bound on the bias in the $\mathrm{Cs}_{2} \mathrm{CO}_{3}$ method of its measurements for each oxide of interest value is also provided at a 95\% confidence. For those oxides present in ARG-1 at concentrations greater than or equal to $0.1 \mathrm{wt} \%$, only $\mathrm{B}_{2} \mathrm{O}_{3}$ has a bias bound (its value is $9.84 \%$ ) that is larger than $4.41 \%$ (the value for $\mathrm{MnO}$ ). Thus, only $\mathrm{B}_{2} \mathrm{O}_{3}$ has a bias bound that is larger than $5 \%$. The low boron bias results from boron volatilization at the $1050{ }^{\circ} \mathrm{C}$ fusion conditions used to enable the $\mathrm{Cs}_{2} \mathrm{CO}_{3}$ method to completely attack large wafers of glass.

\subsection{References}

[1] Bovan, P.L. SME Isolok Acceptance Testing. Savannah River Site, Aiken, SC, 29808 : s.n., 2010. HLW-DWPF-TTR-2010-0036.

[2] Hera, K.R. “Task Technical and Quality Assurance Plan for SME Isolok Acceptance Testing Requirements,” SRNL-RP-2011-00145, Revision 0, 2011.

[3] Hera, K.R., C.J. Coleman, and T.B. Edwards, "Isolok Valve Acceptance Testing for DWPF SME Sampling Process,” SRNL-STI-2011-00693, Revision 0, November 2011.

[4] Edwards, T.B. "Sampling and Analytical Plan Supporting the SME Acceptance Testing of the Isolok Sampler,” SRNL-RP-2011-00294, Revision 0, 2011.

[5] Coleman, C.J., T.B. Edwards, and D.R. Click, "SRNL Evaluation of Sub-Sampling Precision and Accuracy of DWPF Slurry Mix Evaporator Simulant,” WSRC-TR-2004-00339, Revision 0, August 19, 2004.

[6] Coleman, C.J. and T.B. Edwards, "Phase II of a Six Sigma Initiative to Study DWPF SME Analytical Turnaround Times: SRNL's Evaluation of Carbonate-Based Dissolution Methods," WSRC-TR-2005-00396, Revision 0, October 5, 2005.

[7] Coleman, C.J. and T.B. Edwards, "Phase III of a Six Sigma Initiative to Study DWPF SME Analytical Turnaround Times: SRNL Shielded Cell Tests of Large Volume Sub-Sampling of Sludge Batch 4”, WSRC-TR-2007-00274, Revision 0, February 19, 2008.

[8] Bricker, J.M., "Technical Task Request: Development of Statistical Plans and Evaluation of Analyses for Cesium Carbonate Acceptance,” HLW-DWPF-TTR-2010-00025, May 28, 2010. 
[9] Edwards, T.B. and C.J. Coleman, “Task Technical and Quality Assurance Plan: Development of Statistical Plans and Evaluation of Analyses for Cesium Carbonate Acceptance,” SRNLRP-2010-01086, July 2010.

[10] Edwards, T.B., "Cesium Carbonate Dissolution as an Alternative to Mixed Acid Dissolution,” SRNL-TR-2011-00282, October 2011.

[11] SAS Institute, Inc., JMP Version 7.0.2, SAS Institute, Inc., Cary NC, 1989-2007. 
5.0 Appendix

Supplemental Tables and Exhibits 
Table A1. ARG-1 Measured Oxide Concentrations (part 1)

\begin{tabular}{|c|c|c|c|c|c|c|c|c|c|c|c|c|c|c|c|c|}
\hline Test & \begin{tabular}{|c|} 
Type of \\
Material
\end{tabular} & \begin{tabular}{|c|}
$\begin{array}{c}\text { Preparation } \\
\text { Block }\end{array}$ \\
\end{tabular} & $\begin{array}{c}\text { Preparation } \\
\text { Sequence } \\
\end{array}$ & $\begin{array}{c}\text { Analytical } \\
\text { Block }\end{array}$ & \begin{tabular}{|c|} 
Analytical \\
Sequence \\
\end{tabular} & Sample ID & $\begin{array}{l}\mathrm{Al2O3} \\
(\mathrm{wt} \%)\end{array}$ & $\begin{array}{l}\mathrm{B2O3} \\
(\mathrm{wt} \%)\end{array}$ & $\begin{array}{c}\mathrm{BaO} \\
(\mathrm{wt} \%)\end{array}$ & $\begin{array}{c}\mathrm{CaO} \\
(\mathrm{wt} \%)\end{array}$ & $\begin{array}{l}\text { Cr2O3 } \\
(w t \%)\end{array}$ & $\begin{array}{c}\mathrm{CuO} \\
(\mathrm{wt} \%)\end{array}$ & $\begin{array}{l}\text { Fe2O3 } \\
(w t \%)\end{array}$ & $\begin{array}{c}\text { K2O } \\
(w t \%)\end{array}$ & $\begin{array}{c}\mathrm{Li2O} \\
(\mathrm{wt} \%)\end{array}$ & $\begin{array}{c}\text { MgO } \\
(w t \%)\end{array}$ \\
\hline Phase 1 & ARG-1 & 1 & 1 & 1 & 1 & ARG-1B11 & 4.535 & 8.211 & 0.090 & 1.455 & 0.097 & 0.020 & 13.954 & 2.734 & $\begin{array}{l}3.186 \\
\end{array}$ & 0.857 \\
\hline \begin{tabular}{|l|} 
Phase 1 \\
\end{tabular} & ARG-1 & 1 & 15 & 1 & 15 & ARG-1B12 & 4.554 & 8.114 & 0.090 & 1.455 & 0.096 & 0.020 & 13.940 & 2.662 & 3.186 & 0.859 \\
\hline Phase 1 & ARG-1 & 1 & 29 & 1 & 29 & ARG-1B13 & 4.705 & 7.857 & 0.092 & 1.483 & 0.095 & 0.025 & 14.011 & 2.626 & 3.208 & 0.864 \\
\hline \begin{tabular}{|l|} 
Phase 1 \\
\end{tabular} & ARG-1 & 2 & 1 & 2 & 1 & ARG-1B21 & 4.667 & 7.760 & 0.090 & 1.455 & 0.100 & 0.017 & 14.011 & 2.686 & 3.251 & 0.869 \\
\hline \begin{tabular}{|l|} 
Phase 1 \\
\end{tabular} & ARG-1 & 2 & 12 & 2 & 15 & ARG-1B22 & 4.799 & $\begin{array}{l}7.567 \\
\end{array}$ & 0.092 & 1.483 & 0.097 & 0.018 & 14.054 & 2.445 & 3.251 & 0.876 \\
\hline Phase 1 & ARG-1 & 2 & 23 & 2 & 29 & ARG-1B23 & 4.743 & 7.824 & 0.094 & 1.483 & 0.099 & 0.023 & 13.997 & 2.542 & 3.251 & 0.869 \\
\hline Phase 1 & ARG-1 & 3 & 1 & 3 & 1 & ARG-1B31 & 4.686 & 8.468 & 0.092 & 1.469 & 0.104 & 0.021 & 14.025 & 2.662 & 3.251 & 0.871 \\
\hline \begin{tabular}{|l|} 
Phase 1 \\
\end{tabular} & ARG-1 & 3 & 12 & 3 & 15 & ARG-1B32 & 4.724 & 7.953 & 0.091 & 1.469 & 0.096 & 0.022 & \begin{tabular}{|l|}
13.897 \\
\end{tabular} & 2.530 & 3.208 & 0.866 \\
\hline Phase 1 & ARG-1 & 3 & 23 & 3 & 29 & ARG-1B33 & 4.856 & 8.243 & 0.093 & 1.497 & 0.101 & 0.020 & 14.297 & 2.746 & 3.315 & 0.887 \\
\hline Phase 1 & ARG-1 & 4 & 1 & 4 & 1 & ARG-1B41 & 4.648 & 7.985 & 0.090 & 1.455 & 0.096 & 0.022 & 14.011 & \begin{tabular}{|l|}
2.746 \\
\end{tabular} & 3.229 & 0.864 \\
\hline Phase 1 & ARG-1 & 4 & 7 & 4 & 15 & ARG-1B42 & 4.591 & 8.082 & 0.090 & 1.441 & 0.096 & 0.021 & 13.968 & 2.855 & 3.208 & 0.861 \\
\hline Phase 1 & ARG-1 & 4 & 13 & 4 & 29 & ARG-1B43 & 4.667 & 7.857 & 0.090 & $\mid 1.427$ & 0.094 & 0.022 & \begin{tabular}{|l|}
13.997 \\
\end{tabular} & 2.638 & 3.229 & 0.859 \\
\hline \begin{tabular}{|l|} 
Phase 1 \\
\end{tabular} & ARG-1 & 2 & 1 & 5 & 1 & ARG-1B21 & 4.610 & 7.760 & 0.089 & 1.469 & 0.097 & 0.017 & 13.839 & 2.746 & 3.272 & 0.847 \\
\hline Phase 1 & ARG-1 & 2 & 12 & 5 & 15 & ARG-1B22 & 4.270 & 7.309 & 0.083 & 1.367 & 0.090 & 0.018 & 12.867 & 2.494 & 3.036 & 0.789 \\
\hline Phase 1 & ARG-1 & 2 & 23 & 5 & 29 & ARG-1B23 & 4.629 & 7.728 & 0.091 & 1.441 & 0.097 & 0.023 & 14.011 & 2.674 & 3.208 & 0.854 \\
\hline Phase 1 & ARG-1 & 3 & 1 & 6 & 1 & ARG-1B31 & 4.629 & 7.953 & 0.089 & 1.441 & 0.096 & 0.021 & 13.968 & 2.698 & 3.165 & 0.837 \\
\hline Phase 1 & ARG-1 & 3 & 12 & 6 & 15 & ARG-1B32 & 4.554 & 7.953 & 0.087 & 1.427 & 0.095 & 0.022 & 13.668 & 2.626 & 3.100 & 0.823 \\
\hline Phase 1 & ARG-1 & 3 & 23 & 6 & 29 & ARG-1B33 & 4.384 & 7.728 & 0.085 & 1.387 & 0.090 & 0.020 & 13.196 & 2.638 & 3.122 & 0.794 \\
\hline Phase 2 & ARG-1 & 1 & 1 & 1 & 1 & ARG-1B11 & 4.762 & 7.985 & 0.100 & 1.469 & 0.100 & 0.009 & 14.297 & 2.783 & 3.186 & 0.856 \\
\hline Phase 2 & ARG-1 & 1 & 15 & 1 & 15 & ARG-1B12 & 4.780 & 8.018 & 0.099 & 1.483 & 0.100 & 0.002 & 14.583 & 2.783 & 3.229 & 0.872 \\
\hline Phase 2 & ARG-1 & 1 & 29 & 1 & 29 & ARG-1B13 & 4.346 & 7.052 & 0.090 & 1.364 & 0.090 & 0.006 & 13.153 & 2.361 & 2.906 & 0.784 \\
\hline Phase 2 & ARG-1 & 2 & 1 & 2 & 1 & ARG-1B21 & 4.365 & 7.631 & 0.091 & 1.321 & 0.092 & 0.002 & 13.239 & 2.674 & 3.014 & 0.799 \\
\hline Phase 2 & ARG-1 & 2 & 12 & 2 & 15 & ARG-1B22 & 4.573 & 8.114 & 0.098 & \begin{tabular}{|l|}
1.427 \\
\end{tabular} & 0.094 & 0.003 & 13.940 & 2.807 & $\begin{array}{l}3.186 \\
\end{array}$ & 0.841 \\
\hline Phase 2 & ARG-1 & 2 & 23 & 2 & 29 & ARG-1B23 & 4.610 & 8.146 & 0.097 & 1.441 & 0.094 & 0.003 & 13.954 & 2.939 & 3.251 & 0.842 \\
\hline Phase 2 & ARG-1 & 3 & 1 & 3 & 1 & ARG-1B31 & 4.403 & 7.985 & 0.085 & 1.382 & 0.094 & 0.007 & 13.296 & 2.614 & 3.057 & 0.808 \\
\hline Phase 2 & ARG-1 & 3 & 12 & 3 & 15 & ARG-1B32 & 4.610 & 8.243 & 0.098 & 1.483 & 0.096 & 0.009 & 13.997 & 2.903 & 3.208 & 0.852 \\
\hline Phase 2 & ARG-1 & 3 & 23 & 3 & 29 & ARG-1B33 & 4.743 & 8.275 & 0.098 & 1.483 & 0.099 & 0.003 & 14.226 & 2.915 & 3.294 & 0.867 \\
\hline Phase 2 & ARG-1 & 4 & 1 & 4 & 1 & ARG-1B41 & 4.421 & 7.696 & 0.087 & 1.391 & 0.094 & 0.002 & 13.382 & 2.518 & 3.014 & 0.798 \\
\hline Phase 2 & ARG-1 & 4 & 7 & 4 & 15 & ARG-1B42 & 4.535 & 7.631 & 0.097 & 1.455 & 0.096 & 0.003 & 13.839 & 2.674 & $\begin{array}{l}3.100 \\
\end{array}$ & 0.826 \\
\hline Phase 2 & ARG-1 & 4 & 13 & 4 & 29 & ARG-1B43 & 4.648 & 7.792 & 0.091 & 1.455 & 0.103 & 0.003 & 14.140 & 2.734 & 3.165 & 0.842 \\
\hline Phase 2 & ARG-1 & 2 & 1 & 5 & 1 & ARG-1B21 & 4.573 & 8.082 & 0.097 & 1.427 & 0.096 & 0.008 & 13.911 & 2.674 & 3.143 & 0.837 \\
\hline Phase 2 & ARG-1 & 2 & 12 & 5 & 15 & ARG-1B22 & 4.667 & 8.114 & 0.099 & 1.413 & 0.096 & 0.003 & 14.068 & 2.722 & 3.165 & 0.844 \\
\hline Phase 2 & ARG-1 & 2 & 23 & 5 & 29 & ARG-1B23 & 4.440 & 7.438 & 0.093 & 1.349 & 0.091 & 0.003 & 13.353 & 2.554 & 3.014 & 0.801 \\
\hline Phase 2 & ARG-1 & 3 & 1 & 6 & 1 & ARG-1B31 & 4.629 & 8.018 & 0.092 & 1.441 & 0.097 & 0.003 & 13.997 & 2.759 & 3.143 & 0.839 \\
\hline Phase 2 & ARG-1 & 3 & 12 & 6 & 15 & ARG-1B32 & 4.667 & 7.857 & 0.097 & 1.441 & 0.098 & 0.003 & 14.083 & 2.783 & 3.165 & 0.847 \\
\hline Phase 2 & ARG-1 & 3 & 23 & 6 & 29 & ARG-1B33 & 4.421 & 7.309 & 0.090 & 1.381 & 0.092 & 0.003 & 13.253 & 2.590 & 3.014 & 0.798 \\
\hline Phase 3 & ARG-1 & 1 & 1 & 1 & 1 & ARG-1B11 & 4.459 & 7.760 & 0.088 & 1.399 & 0.095 & 0.010 & 13.525 & 2.855 & 3.057 & 0.811 \\
\hline \begin{tabular}{|l|} 
Phase 3 \\
\end{tabular} & ARG-1 & 1 & 15 & 1 & 15 & ARG-1B12 & 4.459 & 7.953 & 0.090 & 1.413 & 0.097 & 0.003 & 13.897 & 2.590 & $\begin{array}{l}3.057 \\
\end{array}$ & 0.827 \\
\hline Phase 3 & ARG-1 & 1 & 29 & 1 & 29 & ARG-1B13 & 4.535 & 7.985 & 0.089 & 1.427 & 0.096 & 0.003 & 13.854 & 2.722 & $\begin{array}{l}3.079 \\
\end{array}$ & 0.824 \\
\hline Phase 3 & ARG-1 & 2 & 1 & 2 & 1 & ARG-1B21 & 4.705 & 8.307 & 0.094 & 1.455 & 0.101 & 0.008 & 14.168 & 2.783 & 3.122 & 0.831 \\
\hline Phase 3 & ARG-1 & 2 & 12 & 2 & 15 & ARG-1B22 & 4.516 & 8.050 & 0.091 & 1.469 & 0.097 & 0.003 & 14.068 & 2.614 & 3.057 & 0.823 \\
\hline Phase 3 & ARG-1 & 2 & 23 & 2 & 29 & ARG-1B23 & 4.629 & 7.696 & \begin{tabular}{l|l|}
0.089 \\
\end{tabular} & 1.469 & 0.106 & 0.003 & 14.025 & 2.903 & 3.100 & 0.823 \\
\hline Phase 3 & ARG-1 & 3 & 1 & 3 & 1 & ARG-1B31 & 4.705 & 7.953 & 0.090 & 1.427 & 0.096 & 0.003 & 14.083 & 2.710 & 3.165 & 0.847 \\
\hline Phase 3 & ARG-1 & 3 & 12 & 3 & 15 & ARG-1B32 & 4.686 & 7.824 & 0.092 & 1.455 & 0.103 & 0.003 & 14.226 & 2.867 & 3.143 & 0.857 \\
\hline
\end{tabular}


Table A1. ARG-1 Measured Oxide Concentrations (part 1)

\begin{tabular}{|c|c|c|c|c|c|c|c|c|c|c|c|c|c|c|c|c|}
\hline Test & \begin{tabular}{|c|} 
Type of \\
Material
\end{tabular} & \begin{tabular}{|c|}
$\begin{array}{c}\text { Preparation } \\
\text { Block }\end{array}$ \\
\end{tabular} & $\begin{array}{c}\text { Preparation } \\
\text { Sequence }\end{array}$ & \begin{tabular}{|c|}
$\begin{array}{c}\text { Analytical } \\
\text { Block }\end{array}$ \\
\end{tabular} & \begin{tabular}{|c} 
Analytical \\
Sequence \\
\end{tabular} & Sample ID & $\begin{array}{l}\text { Al2O3 } \\
(\mathbf{w t} \%) \\
\end{array}$ & $\begin{array}{l}\mathrm{B2O3} \\
(\mathrm{wt} \%) \\
\end{array}$ & $\begin{array}{c}\mathrm{BaO} \\
(\mathrm{wt} \%)\end{array}$ & $\begin{array}{c}\mathrm{CaO} \\
(\mathrm{wt} \%)\end{array}$ & $\begin{array}{l}\text { Cr2O3 } \\
(\text { wt } \%) \\
\end{array}$ & $\begin{array}{c}\mathrm{CuO} \\
(\mathrm{wt} \%)\end{array}$ & $\begin{array}{l}\text { Fe2O3 } \\
(w t \%) \\
\end{array}$ & $\begin{array}{c}\text { K2O } \\
(w t \%)\end{array}$ & $\begin{array}{c}\text { Li2O } \\
(w t \%)\end{array}$ & $\begin{array}{c}\text { MgO } \\
(\mathbf{w t} \%) \\
\end{array}$ \\
\hline Phase 3 & ARG-1 & 3 & 23 & 3 & 29 & ARG-1B33 & 4.573 & 7.599 & 0.091 & 1.441 & 0.095 & 0.003 & 13.868 & 2.698 & 3.186 & 0.839 \\
\hline Phase 3 & ARG-1 & 4 & 1 & 4 & 1 & ARG-1B41 & 4.554 & 7.921 & 0.095 & 1.469 & 0.098 & 0.003 & 14.083 & 2.771 & 3.186 & 0.857 \\
\hline Phase 3 & ARG-1 & 4 & 7 & 4 & 14 & ARG-1B42 & 4.591 & 7.857 & 0.095 & 1.427 & 0.098 & 0.003 & 14.011 & 2.674 & 3.143 & 0.847 \\
\hline \begin{tabular}{|l|} 
Phase 3 \\
\end{tabular} & ARG-1 & 4 & 13 & 4 & 26 & ARG-1B43 & 4.535 & 7.535 & 0.093 & 1.427 & 0.095 & 0.003 & 13.968 & 2.843 & 3.122 & 0.842 \\
\hline Phase 3 & ARG-1 & 2 & 1 & 5 & 1 & ARG-1B21 & 4.743 & 8.050 & 0.093 & 1.469 & 0.103 & 0.011 & 14.011 & 2.554 & 3.229 & 0.866 \\
\hline Phase 3 & ARG-1 & 2 & 12 & 5 & 15 & ARG-1B22 & 4.762 & 8.436 & 0.098 & 1.511 & 0.101 & 0.006 & 14.083 & 2.759 & 3.337 & 0.872 \\
\hline Phase 3 & ARG-1 & 2 & 23 & 5 & 29 & ARG-1B23 & 4.762 & 8.758 & 0.093 & 1.539 & 0.116 & 0.017 & 14.054 & 2.831 & 3.229 & 0.866 \\
\hline \begin{tabular}{|l|} 
Phase 3 \\
\end{tabular} & ARG-1 & 3 & 1 & 6 & 1 & ARG-1B31 & 4.743 & $\begin{array}{l}8.146 \\
\end{array}$ & 0.092 & 1.469 & 0.102 & 0.003 & 14.111 & 2.602 & 3.100 & 0.839 \\
\hline Phase 3 & ARG-1 & 3 & 12 & 6 & 15 & ARG-1B32 & 4.629 & 8.404 & 0.093 & 1.497 & 0.111 & 0.003 & 14.154 & 2.710 & 3.079 & 0.837 \\
\hline Phase 3 & ARG-1 & 3 & 23 & 6 & 29 & ARG-1B33 & 4.762 & 8.179 & 0.093 & 1.469 & 0.106 & 0.003 & 13.997 & 2.494 & 3.100 & 0.836 \\
\hline
\end{tabular}


Table A1. ARG-1 Measured Oxide Concentrations (part 2)

\begin{tabular}{|c|c|c|c|c|c|c|c|c|c|c|c|c|c|c|}
\hline Test & \begin{tabular}{|c|} 
Type of \\
Material
\end{tabular} & \begin{tabular}{|c|}
$\begin{array}{c}\text { Preparation } \\
\text { Block }\end{array}$ \\
\end{tabular} & \begin{tabular}{|c|} 
Preparation \\
Sequence
\end{tabular} & \begin{tabular}{|c|}
$\begin{array}{c}\text { Analytical } \\
\text { Block }\end{array}$ \\
\end{tabular} & $\begin{array}{c}\text { Analytical } \\
\text { Sequence }\end{array}$ & Sample ID & $\begin{array}{c}\mathrm{MnO} \\
(\mathrm{wt} \%)\end{array}$ & $\begin{array}{l}\mathrm{Na2O} \\
(\mathrm{wt} \%)\end{array}$ & $\begin{array}{c}\mathrm{NiO} \\
(\mathrm{wt} \%)\end{array}$ & $\begin{array}{c}\mathrm{SiO2} \\
(\mathrm{wt} \%)\end{array}$ & $\begin{array}{c}\mathrm{TiO2} \\
(\mathrm{wt} \%)\end{array}$ & $\begin{array}{c}\mathrm{ZnO} \\
(\mathrm{wt} \%)\end{array}$ & $\begin{array}{l}\mathrm{ZrO2} \\
(\mathrm{wt} \%)\end{array}$ & $\begin{array}{c}\text { Sum of } \\
\text { Oxides (wt } \%) \\
\end{array}$ \\
\hline Phase 1 & ARG-1 & 1 & 1 & 1 & 1 & ARG-1B11 & 1.846 & 11.525 & 1.035 & 48.348 & 1.154 & 0.024 & 0.133 & 99.205 \\
\hline Phase 1 & ARG-1 & 1 & 15 & 1 & 15 & ARG-1B12 & 1.859 & 11.606 & \begin{tabular}{|l|}
1.036 \\
\end{tabular} & 48.348 & 1.151 & 0.025 & 0.133 & 99.135 \\
\hline Phase 1 & ARG-1 & 1 & 29 & 1 & 29 & ARG-1B13 & 1.859 & 11.687 & \begin{tabular}{|l|}
1.026 \\
\end{tabular} & 48.348 & 1.158 & 0.024 & 0.136 & 99.204 \\
\hline Phase 1 & ARG-1 & 2 & 1 & 2 & 1 & ARG-1B21 & 1.885 & 11.633 & \begin{tabular}{|l|}
1.068 \\
\end{tabular} & 48.562 & 1.158 & 0.024 & 0.135 & 99.372 \\
\hline Phase 1 & ARG-1 & 2 & 12 & 2 & 15 & ARG-1B22 & 1.898 & 11.701 & 1.029 & 48.990 & 1.159 & 0.023 & 0.135 & 99.618 \\
\hline Phase 1 & ARG-1 & 2 & 23 & 2 & 29 & ARG-1B23 & 1.898 & 11.755 & $\begin{array}{l}1.055 \\
\end{array}$ & 48.562 & 1.161 & 0.024 & 0.133 & 99.511 \\
\hline \begin{tabular}{|l|} 
Phase 1 \\
\end{tabular} & ARG-1 & 3 & 1 & 3 & 1 & ARG-1B31 & 1.898 & 11.674 & \begin{tabular}{l|}
1.071 \\
\end{tabular} & 48.990 & 1.168 & 0.024 & 0.132 & 100.608 \\
\hline Phase 1 & ARG-1 & 3 & 12 & 3 & 15 & ARG-1B32 & 1.885 & 11.579 & \begin{tabular}{|l|}
1.029 \\
\end{tabular} & 48.348 & 1.149 & 0.023 & 0.130 & 98.999 \\
\hline Phase 1 & ARG-1 & 3 & 23 & 3 & 29 & ARG-1B33 & 1.937 & 11.916 & 1.063 & 50.060 & 1.183 & 0.023 & 0.139 & 102.375 \\
\hline Phase 1 & ARG-1 & 4 & 1 & 4 & 1 & ARG-1B41 & 1.872 & 11.458 & 1.014 & 48.134 & 1.153 & 0.023 & 0.131 & 98.934 \\
\hline Phase 1 & ARG-1 & 4 & 7 & 4 & 15 & ARG-1B42 & 1.859 & 11.458 & $\begin{array}{l}1.007 \\
\end{array}$ & 48.134 & 1.156 & 0.025 & 0.133 & 98.985 \\
\hline Phase 1 & ARG-1 & 4 & 13 & 4 & 29 & ARG-1B43 & 1.859 & 11.525 & 1.005 & 48.134 & 1.156 & 0.024 & 0.133 & 98.717 \\
\hline Phase 1 & ARG-1 & 2 & 1 & 5 & 1 & ARG-1B21 & 1.834 & 11.458 & 1.015 & 47.706 & 1.141 & 0.024 & 0.132 & 98.059 \\
\hline Phase 1 & ARG-1 & 2 & 12 & 5 & 15 & ARG-1B22 & 1.704 & 10.744 & 0.937 & 44.711 & 1.063 & 0.022 & 0.122 & 91.626 \\
\hline Phase 1 & ARG-1 & 2 & 23 & 5 & 29 & ARG-1B23 & 1.859 & 11.714 & 1.015 & 48.348 & 1.154 & 0.025 & 0.132 & 99.004 \\
\hline Phase 1 & ARG-1 & 3 & 1 & 6 & 1 & ARG-1B31 & 1.821 & 11.512 & 1.013 & 47.920 & 1.128 & 0.024 & 0.130 & 98.445 \\
\hline Phase 1 & ARG-1 & 3 & 12 & 6 & 15 & ARG-1B32 & 1.782 & 11.418 & 0.998 & 47.279 & 1.104 & 0.023 & 0.131 & 97.090 \\
\hline Phase 1 & ARG-1 & 3 & 23 & 6 & 29 & ARG-1B33 & 1.730 & 11.121 & 0.973 & 45.781 & 1.071 & 0.022 & 0.128 & 94.270 \\
\hline Phase 2 & ARG-1 & 1 & 1 & 1 & 1 & ARG-1B11 & 1.846 & 11.822 & 1.029 & 49.418 & 1.158 & 0.026 & 0.130 & 100.977 \\
\hline Phase 2 & ARG-1 & 1 & 15 & 1 & 15 & ARG-1B12 & 1.885 & 12.038 & 1.037 & 50.487 & 1.174 & 0.026 & 0.131 & 102.729 \\
\hline Phase 2 & ARG-1 & 1 & 29 & 1 & 29 & ARG-1B13 & 1.691 & 10.878 & 0.944 & 45.139 & 1.058 & 0.023 & 0.117 & 92.003 \\
\hline Phase 2 & ARG-1 & 2 & 1 & 2 & 1 & ARG-1B21 & 1.743 & 10.744 & 0.965 & 44.925 & 1.096 & 0.025 & 0.130 & 92.856 \\
\hline Phase 2 & ARG-1 & 2 & 12 & 2 & 15 & ARG-1B22 & 1.834 & 11.458 & 1.021 & 47.492 & 1.156 & 0.027 & 0.136 & 98.206 \\
\hline Phase 2 & ARG-1 & 2 & 23 & 2 & 29 & ARG-1B23 & 1.846 & 11.647 & 1.014 & 47.706 & 1.173 & 0.025 & 0.143 & 98.933 \\
\hline Phase 2 & ARG-1 & 3 & 1 & 3 & 1 & ARG-1B31 & 1.769 & 10.851 & 0.965 & 45.353 & 1.101 & 0.022 & 0.124 & 93.916 \\
\hline Phase 2 & ARG-1 & 3 & 12 & 3 & 15 & ARG-1B32 & 1.859 & 11.606 & 1.003 & 48.348 & 1.164 & 0.025 & 0.139 & 99.644 \\
\hline Phase 2 & ARG-1 & 3 & 23 & 3 & 29 & ARG-1B33 & 1.898 & 11.795 & 1.026 & 48.990 & 1.181 & 0.026 & 0.133 & 101.051 \\
\hline Phase 2 & ARG-1 & 4 & 1 & 4 & 1 & ARG-1B41 & 1.730 & 10.851 & 0.981 & 45.995 & 1.108 & 0.024 & 0.126 & 94.217 \\
\hline Phase 2 & ARG-1 & 4 & 7 & 4 & 15 & ARG-1B42 & 1.795 & 11.337 & 1.008 & 47.492 & 1.149 & 0.027 & 0.130 & 97.194 \\
\hline Phase 2 & ARG-1 & 4 & 13 & 4 & 29 & ARG-1B43 & 1.834 & 11.593 & 1.017 & 48.776 & 1.166 & 0.022 & 0.139 & 99.520 \\
\hline Phase 2 & ARG-1 & 2 & 1 & 5 & 1 & ARG-1B21 & 1.821 & 11.377 & 1.014 & 47.706 & 1.153 & 0.026 & 0.133 & 98.078 \\
\hline Phase 2 & ARG-1 & 2 & 12 & 5 & 15 & ARG-1B22 & 1.821 & 11.458 & 1.023 & 48.348 & 1.156 & 0.025 & 0.136 & 99.158 \\
\hline Phase 2 & ARG-1 & 2 & 23 & 5 & 29 & ARG-1B23 & 1.730 & 10.932 & \begin{tabular}{l|l|}
0.968 \\
\end{tabular} & 45.781 & 1.093 & 0.026 & 0.126 & 93.793 \\
\hline Phase 2 & ARG-1 & 3 & 1 & 6 & 1 & ARG-1B31 & 1.821 & 11.350 & 1.004 & 47.492 & 1.156 & 0.023 & 0.133 & 97.996 \\
\hline Phase 2 & ARG-1 & 3 & 12 & 6 & 15 & ARG-1B32 & 1.821 & 11.377 & 1.009 & 47.920 & 1.158 & 0.027 & 0.131 & 98.482 \\
\hline Phase 2 & ARG-1 & 3 & 23 & 6 & 29 & ARG-1B33 & 1.730 & 10.744 & 0.954 & 45.139 & 1.089 & 0.025 & 0.121 & 92.754 \\
\hline Phase 3 & ARG-1 & 1 & 1 & 1 & 1 & ARG-1B11 & 1.756 & 10.824 & 1.000 & 48.134 & 1.149 & 0.028 & 0.132 & 97.084 \\
\hline Phase 3 & ARG-1 & 1 & 15 & 1 & 15 & ARG-1B12 & 1.795 & 10.986 & 1.042 & 49.204 & 1.169 & 0.025 & 0.135 & 98.743 \\
\hline Phase 3 & ARG-1 & 1 & 29 & 1 & 29 & ARG-1B13 & 1.795 & 10.986 & 1.008 & 48.990 & 1.176 & 0.024 & 0.138 & 98.731 \\
\hline Phase 3 & ARG-1 & 2 & 1 & 2 & 1 & ARG-1B21 & 1.808 & 11.701 & 1.051 & 49.204 & 1.184 & 0.025 & 0.138 & 100.683 \\
\hline Phase 3 & ARG-1 & 2 & 12 & 2 & 15 & ARG-1B22 & 1.795 & $\begin{array}{l}11.647 \\
\end{array}$ & \begin{tabular}{l|}
1.029 \\
\end{tabular} & 48.562 & 1.168 & 0.024 & 0.139 & 99.151 \\
\hline Phase 3 & ARG-1 & 2 & 23 & 2 & 29 & ARG-1B23 & 1.782 & 11.782 & $\begin{array}{l}0.985 \\
\end{array}$ & 48.776 & 1.173 & 0.021 & 0.135 & 99.497 \\
\hline Phase 3 & ARG-1 & 3 & 1 & 3 & 1 & ARG-1B31 & 1.834 & 11.525 & 1.010 & 49.204 & 1.169 & 0.025 & 0.133 & 99.980 \\
\hline Phase 3 & ARG-1 & 3 & 12 & 3 & 15 & ARG-1B32 & 1.859 & 11.593 & 1.033 & 49.204 & 1.168 & 0.026 & 0.132 & 100.272 \\
\hline
\end{tabular}


Table A1. ARG-1 Measured Oxide Concentrations (part 2)

\begin{tabular}{|c|c|c|c|c|c|c|c|c|c|c|c|c|c|c|}
\hline Test & \begin{tabular}{|c|}
$\begin{array}{c}\text { Type of } \\
\text { Material }\end{array}$ \\
\end{tabular} & \begin{tabular}{|c|}
$\begin{array}{c}\text { Preparation } \\
\text { Block }\end{array}$ \\
\end{tabular} & \begin{tabular}{|c|} 
Preparation \\
Sequence
\end{tabular} & \begin{tabular}{|c|}
$\begin{array}{c}\text { Analytical } \\
\text { Block }\end{array}$ \\
\end{tabular} & \begin{tabular}{|c|} 
Analytical \\
Sequence
\end{tabular} & Sample ID & $\begin{array}{c}\mathrm{MnO} \\
(\mathrm{wt} \%)\end{array}$ & $\begin{array}{l}\text { Na2O } \\
(w t \%)\end{array}$ & $\begin{array}{c}\mathrm{NiO} \\
(\mathrm{wt} \%)\end{array}$ & $\begin{array}{c}\mathrm{SiO2} \\
(\mathbf{w t} \%)\end{array}$ & $\begin{array}{c}\mathrm{TiO2} \\
(\mathbf{w t} \%)\end{array}$ & $\begin{array}{c}\mathrm{ZnO} \\
(\mathrm{wt} \%)\end{array}$ & $\begin{array}{c}\mathrm{ZrO2} \\
(\mathrm{wt} \%)\end{array}$ & \begin{tabular}{|c|} 
Sum of \\
Oxides (wt $\%)$ \\
\end{tabular} \\
\hline Phase 3 & ARG-1 & 3 & 23 & 3 & 29 & ARG-1B33 & 1.821 & 11.593 & 1.007 & 48.562 & 1.156 & 0.024 & 0.133 & 98.688 \\
\hline \begin{tabular}{|l|} 
Phase 3 \\
\end{tabular} & ARG-1 & 4 & 1 & 4 & 1 & ARG-1B41 & 1.872 & 11.593 & 1.029 & 48.562 & 1.161 & 0.024 & 0.128 & 99.406 \\
\hline \begin{tabular}{|l|} 
Phase 3 \\
\end{tabular} & ARG-1 & 4 & 7 & 4 & 14 & ARG-1B42 & 1.846 & $\begin{array}{l}11.579 \\
\end{array}$ & 1.040 & 48.348 & 1.144 & 0.026 & 0.127 & 98.857 \\
\hline \begin{tabular}{|l|} 
Phase 3 \\
\end{tabular} & ARG-1 & 4 & 13 & 4 & 26 & ARG-1B43 & 1.834 & 11.458 & 1.007 & 48.134 & 1.136 & 0.025 & 0.128 & 98.184 \\
\hline \begin{tabular}{|l|} 
Phase 3 \\
\end{tabular} & ARG-1 & 2 & 1 & 5 & 1 & ARG-1B21 & 1.885 & 11.391 & 1.029 & 48.348 & 1.151 & 0.025 & 0.131 & 99.089 \\
\hline Phase 3 & ARG-1 & 2 & 12 & 5 & 15 & ARG-1B22 & 1.885 & 11.876 & 1.049 & 48.990 & 1.156 & 0.025 & 0.132 & 101.077 \\
\hline Phase 3 & ARG-1 & 2 & 23 & 5 & 29 & ARG-1B23 & 1.885 & 11.377 & 1.050 & 48.562 & 1.163 & 0.023 & 0.129 & 100.452 \\
\hline \begin{tabular}{|l|} 
Phase 3 \\
\end{tabular} & ARG-1 & 3 & 1 & 6 & 1 & ARG-1B31 & 1.821 & $\begin{array}{l}11.539 \\
\end{array}$ & 1.052 & 48.776 & 1.164 & 0.025 & 0.127 & 99.712 \\
\hline Phase 3 & ARG-1 & 3 & 12 & 6 & 15 & ARG-1B32 & 1.821 & 11.579 & 1.084 & 48.990 & 1.169 & 0.024 & 0.136 & 100.321 \\
\hline \begin{tabular}{|l|} 
Phase 3 \\
\end{tabular} & ARG-1 & 3 & 23 & 6 & 29 & ARG-1B33 & 1.808 & \begin{tabular}{l|l|}
11.647 \\
\end{tabular} & 1.069 & 48.562 & 1.163 & 0.024 & 0.128 & 99.437 \\
\hline
\end{tabular}




\section{Table A2. Reference Oxide Composition for ARG-1}

\begin{tabular}{|c|c|}
\hline Oxide & $\begin{array}{c}\text { Targeted } \\
\text { wt } \%\end{array}$ \\
\hline $\mathrm{Al} 2 \mathrm{O} 3(\mathrm{wt} \%)$ & 4.73 \\
\hline B2O3 (wt\%) & 8.67 \\
\hline $\mathrm{BaO}(\mathrm{wt} \%)$ & 0.09 \\
\hline $\mathrm{CaO}(\mathrm{wt} \%)$ & 1.43 \\
\hline Cr2O3 (wt\%) & 0.09 \\
\hline $\mathrm{CuO}$ (wt\%) & 0 \\
\hline Fe2O3 (wt\%) & 14 \\
\hline $\mathrm{K} 2 \mathrm{O}(\mathrm{wt} \%)$ & 2.71 \\
\hline Li2O (wt\%) & 3.21 \\
\hline $\mathrm{MgO}(\mathrm{wt} \%)$ & 0.86 \\
\hline $\mathrm{MnO}(\mathrm{wt} \%)$ & 1.89 \\
\hline $\mathrm{Na} 2 \mathrm{O}(\mathrm{wt} \%)$ & 11.5 \\
\hline $\mathrm{NiO}$ (wt\%) & 1.05 \\
\hline $\mathrm{SiO} 2$ (wt\%) & 47.9 \\
\hline TiO2 (wt\%) & 1.15 \\
\hline $\mathrm{ZnO}(\mathrm{wt} \%)$ & 0.02 \\
\hline $\mathrm{ZrO} 2$ (wt\%) & 0.13 \\
\hline Sum of Oxides (wt\%) & 99.43 \\
\hline
\end{tabular}


Exhibit A1. Variability Chart for ARG-1 Oxide Measurements

Analyte $=\mathrm{Al2O} 3(\mathrm{wt} \%)$

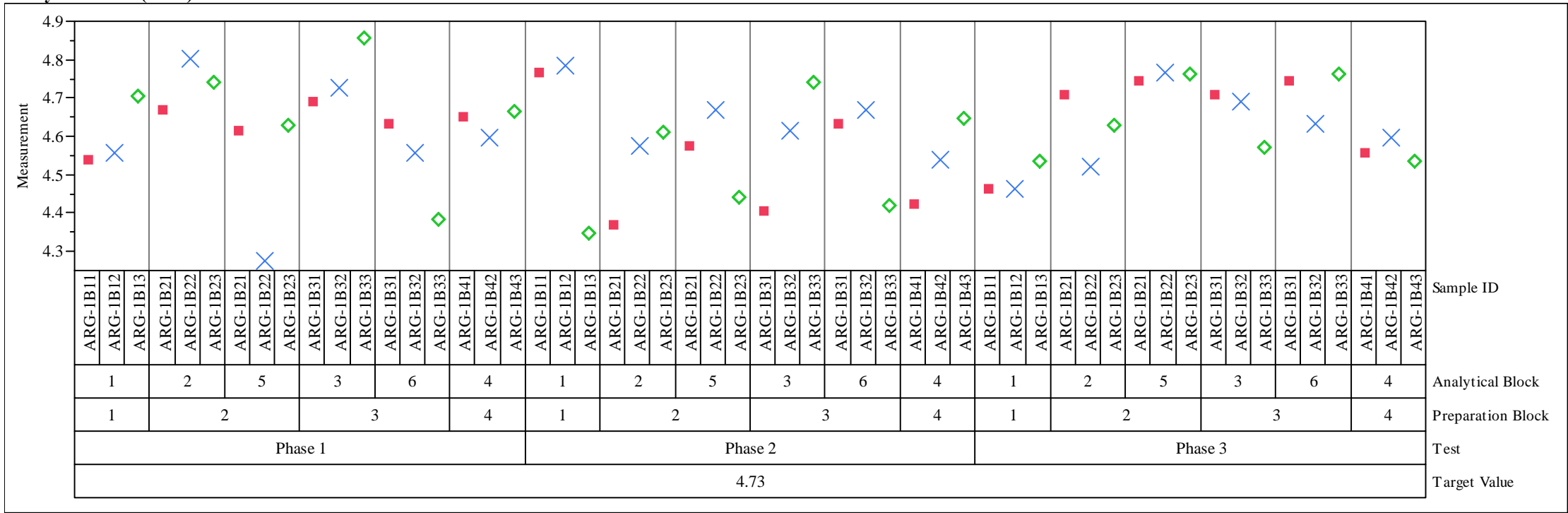

Analyte $=\mathbf{B 2 O 3}(\mathrm{wt} \%)$

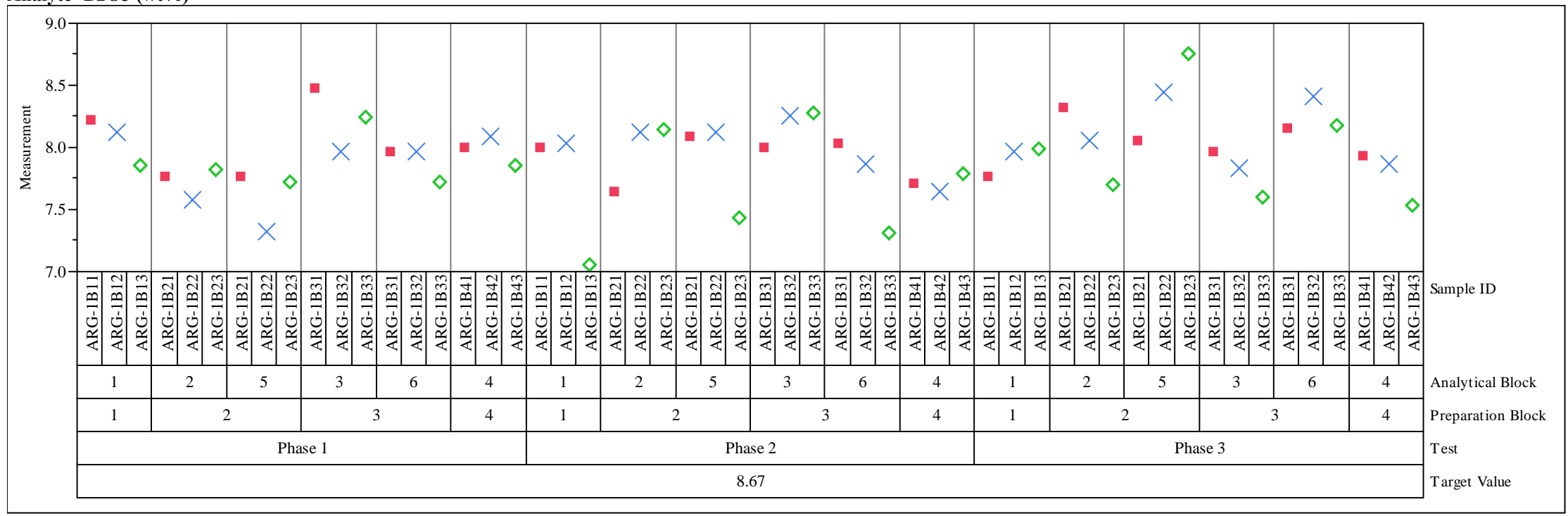


Exhibit A1. Variability Chart for ARG-1 Oxide Measurements

Analyte $=\mathrm{BaO}(\mathrm{wt} \%)$

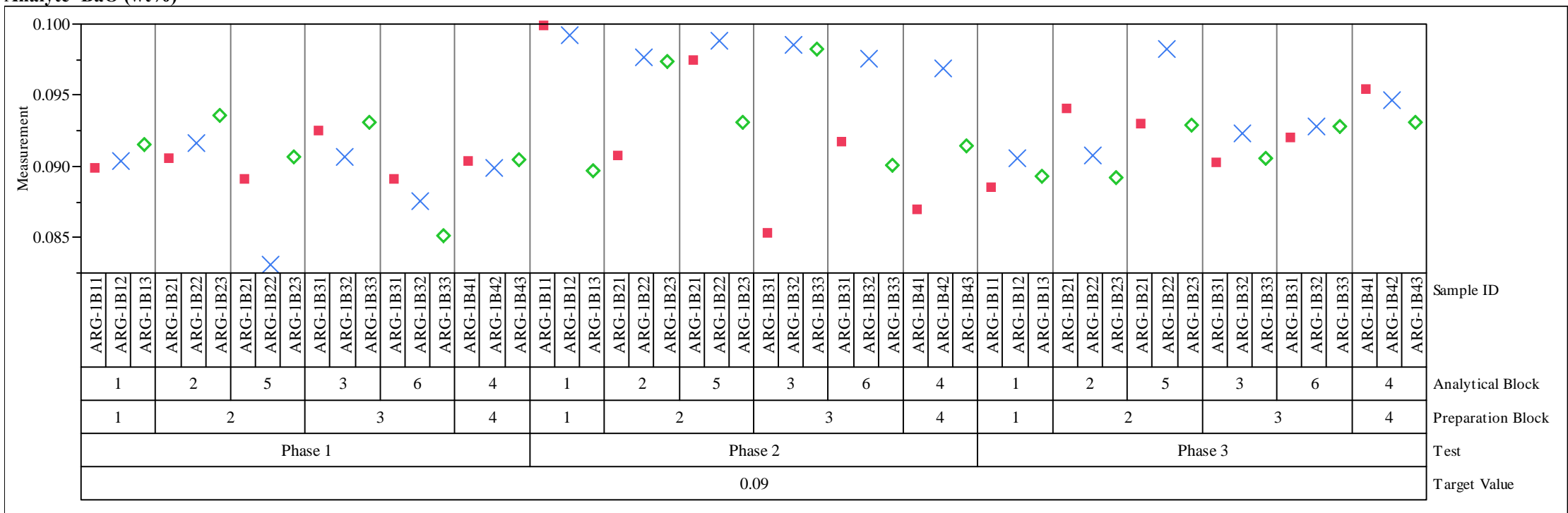

Analyte $=\mathbf{C a O}($ wt $\%)$

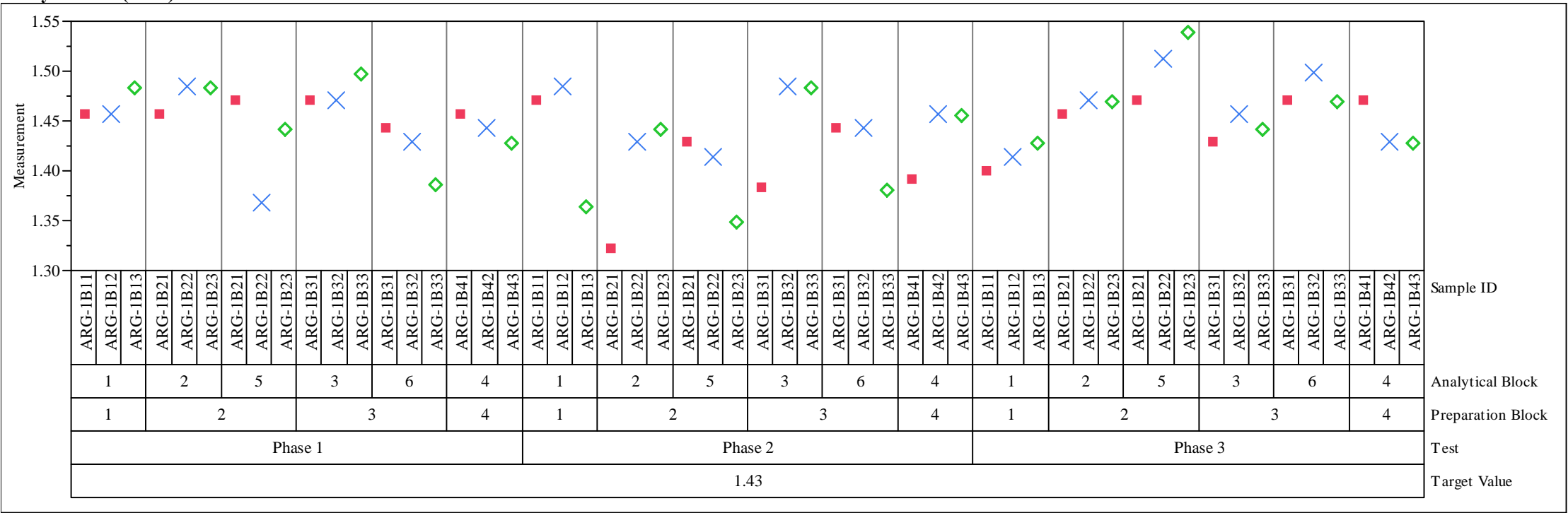


Exhibit A1. Variability Chart for ARG-1 Oxide Measurements

Analyte $=\mathrm{Cr} 2 \mathrm{O} 3(\mathrm{wt} \%)$

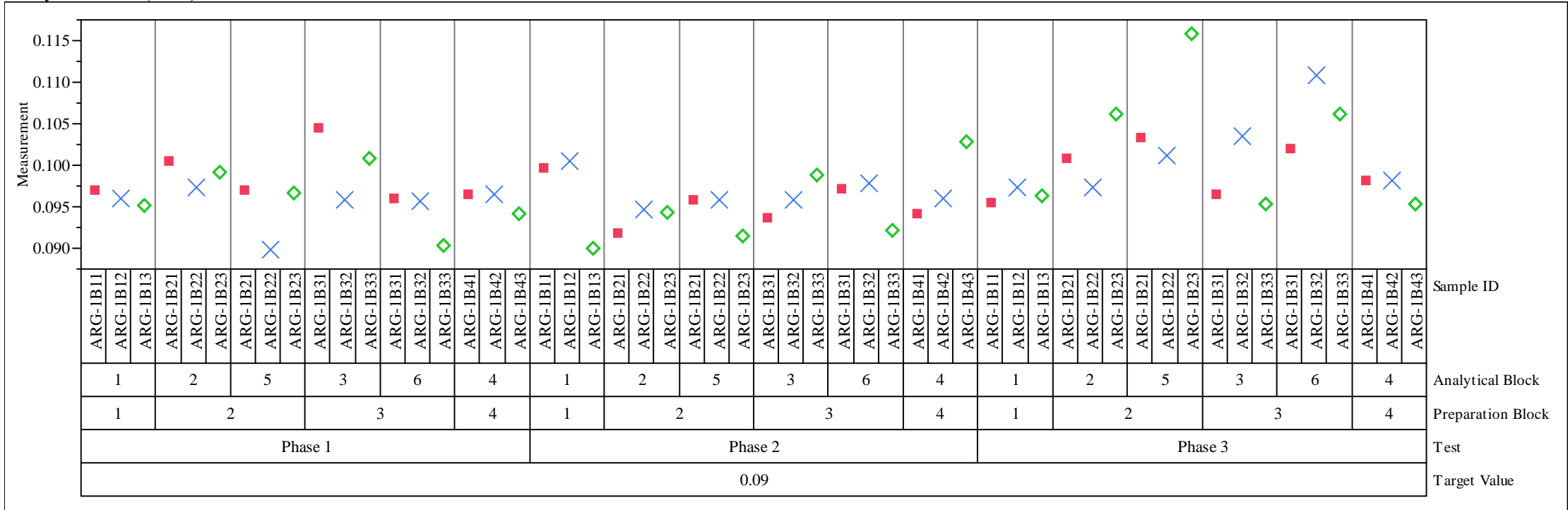

Analyte $=\mathrm{CuO}(\mathrm{wt} \%)$

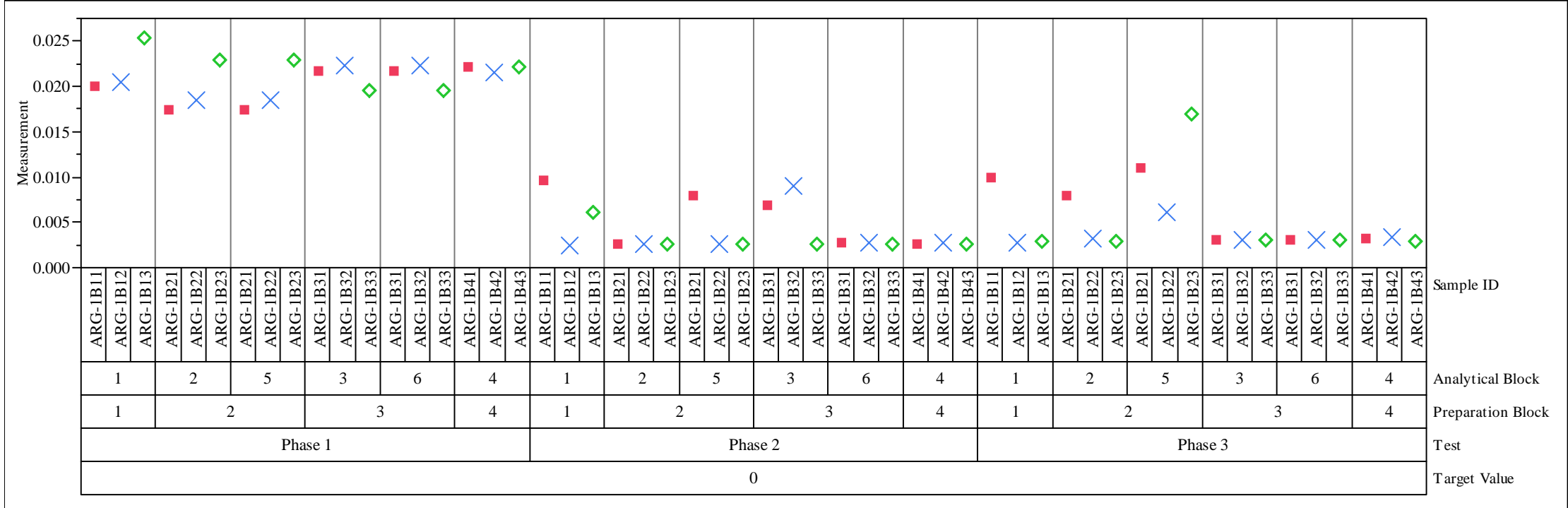


Exhibit A1. Variability Chart for ARG-1 Oxide Measurements

Analyte $=\mathrm{Fe} 2 \mathrm{O} 3(\mathrm{wt} \%)$

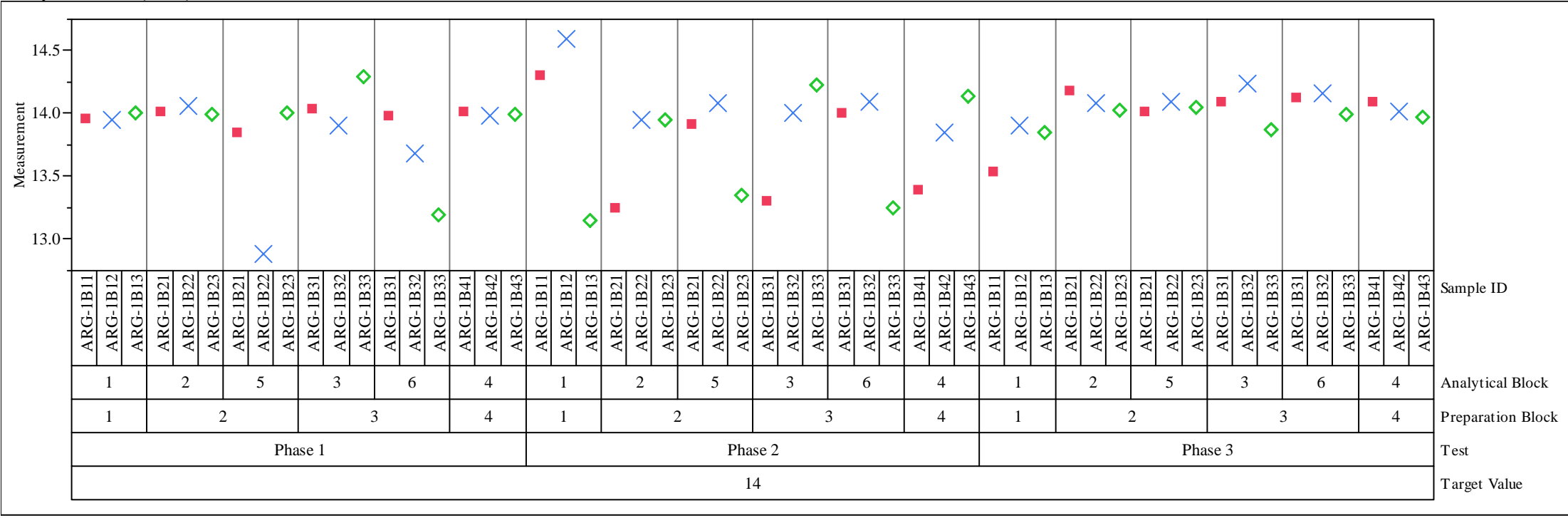

Analyte $=\mathrm{K} 20(\mathrm{wt} \%)$

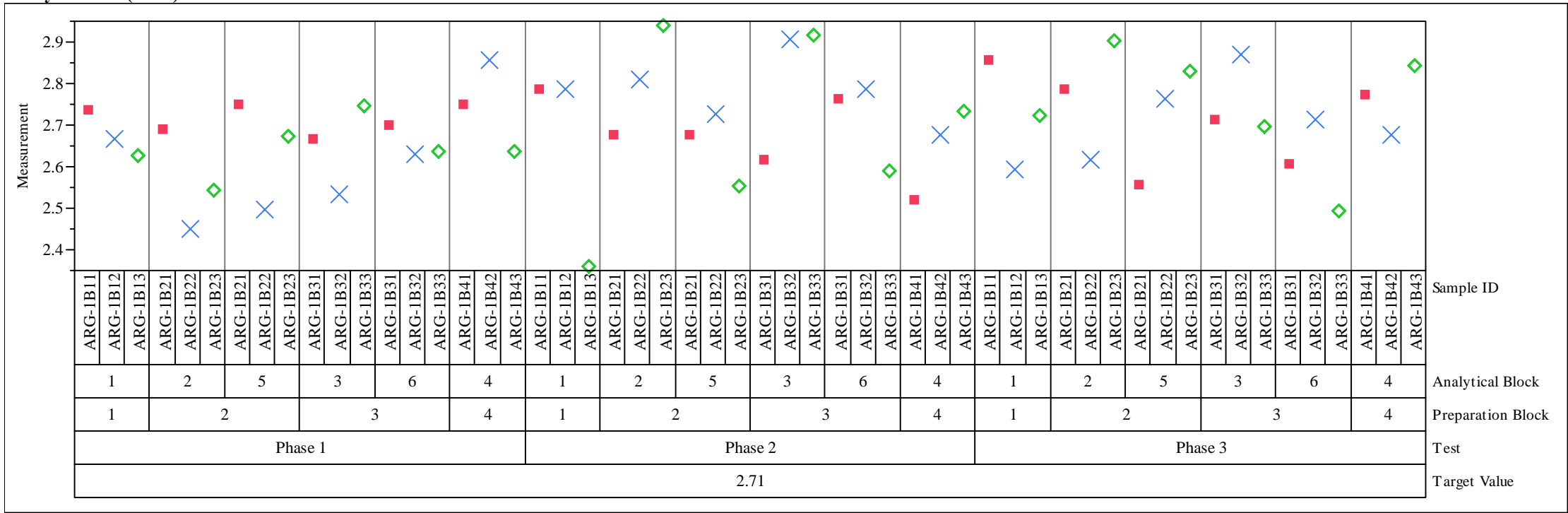


Exhibit A1. Variability Chart for ARG-1 Oxide Measurements

Analyte $=\mathrm{Li2O}(\mathrm{wt} \%)$

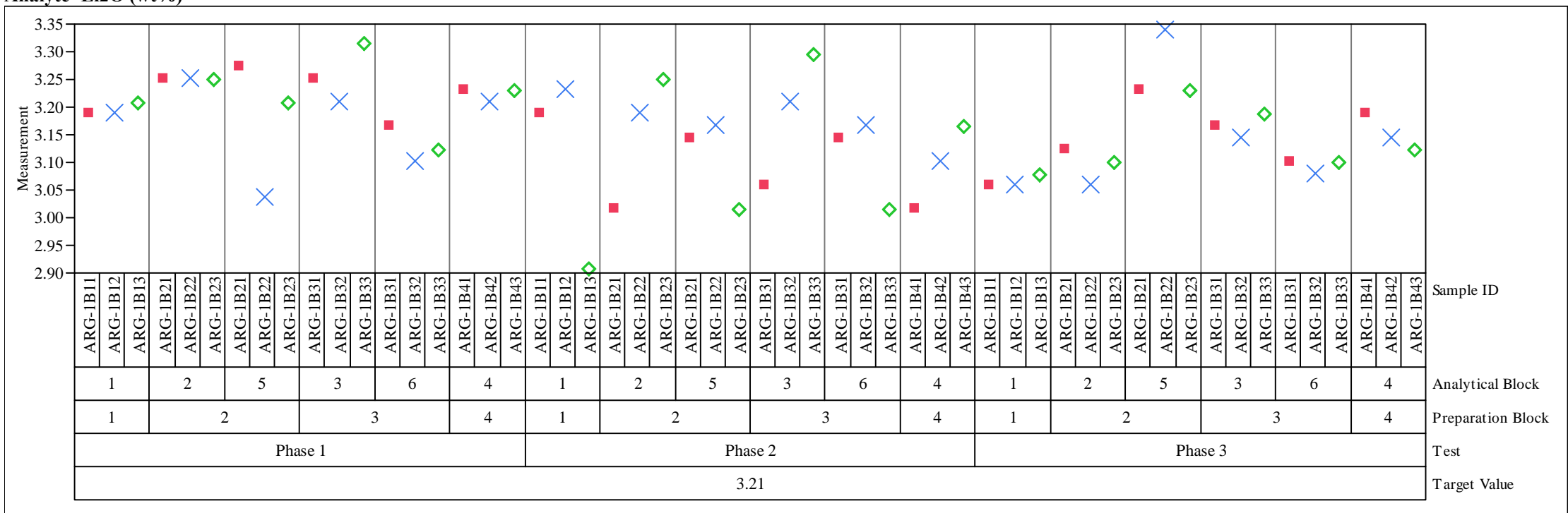

Analyte $=\mathrm{MgO}(\mathrm{wt} \%)$

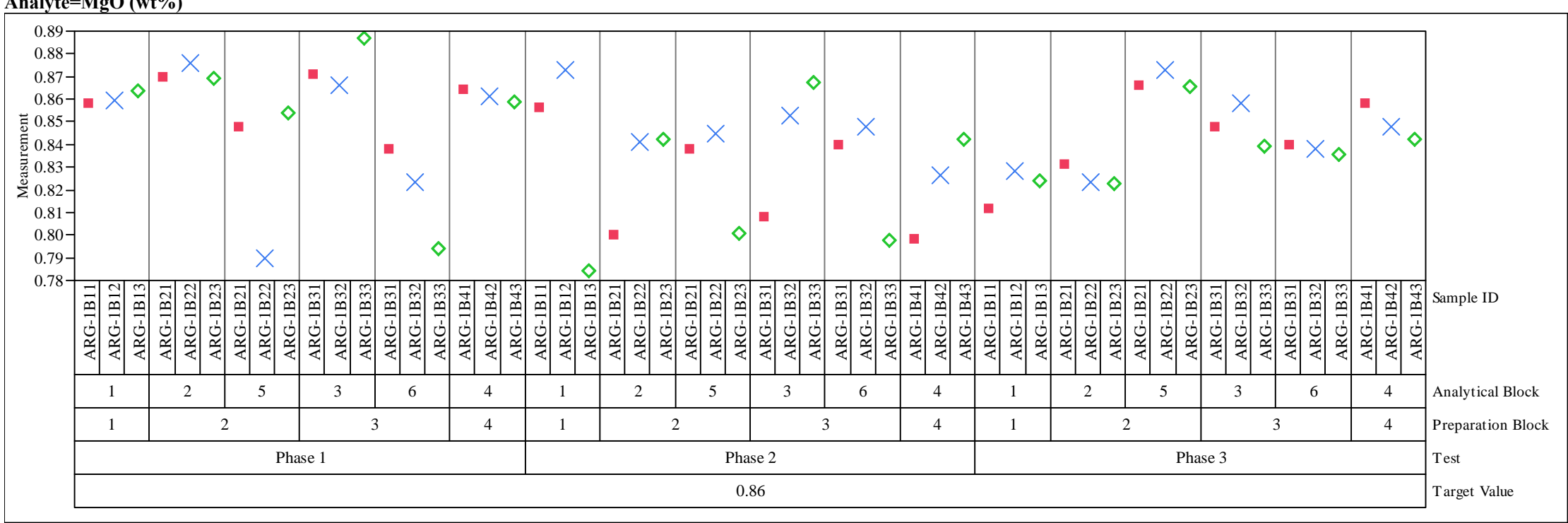


Exhibit A1. Variability Chart for ARG-1 Oxide Measurements

Analyte $=\mathrm{MnO}(\mathrm{wt} \%)$
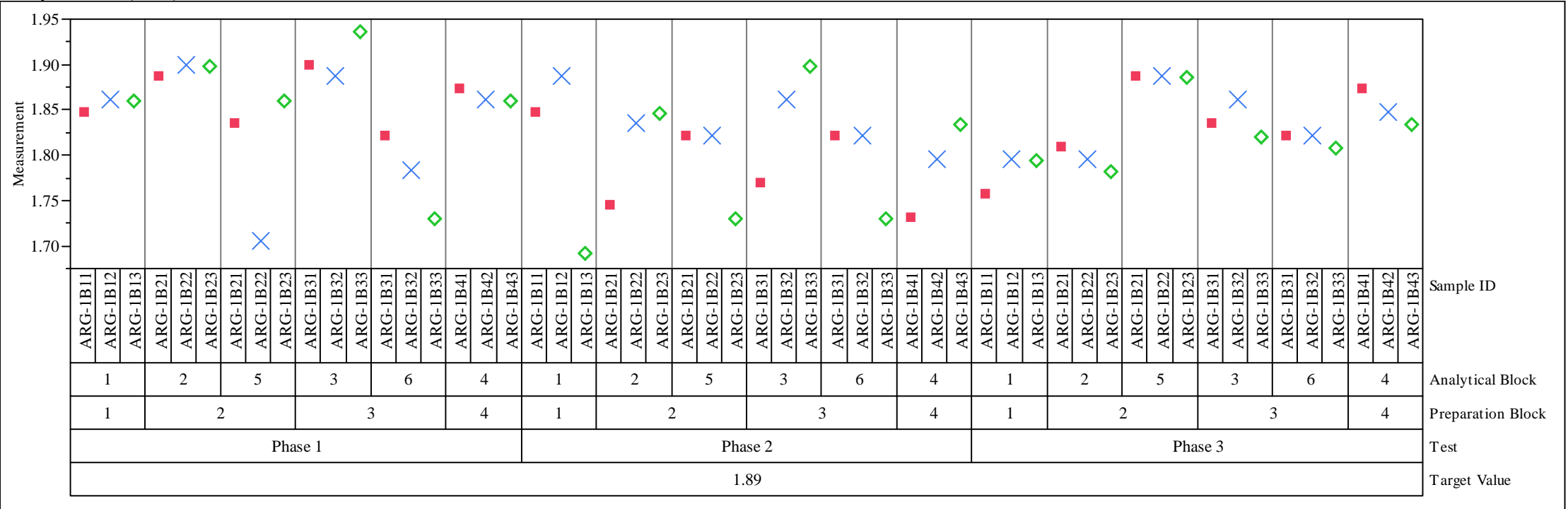

Analyte $=\mathrm{Na2O}(\mathrm{wt} \%)$

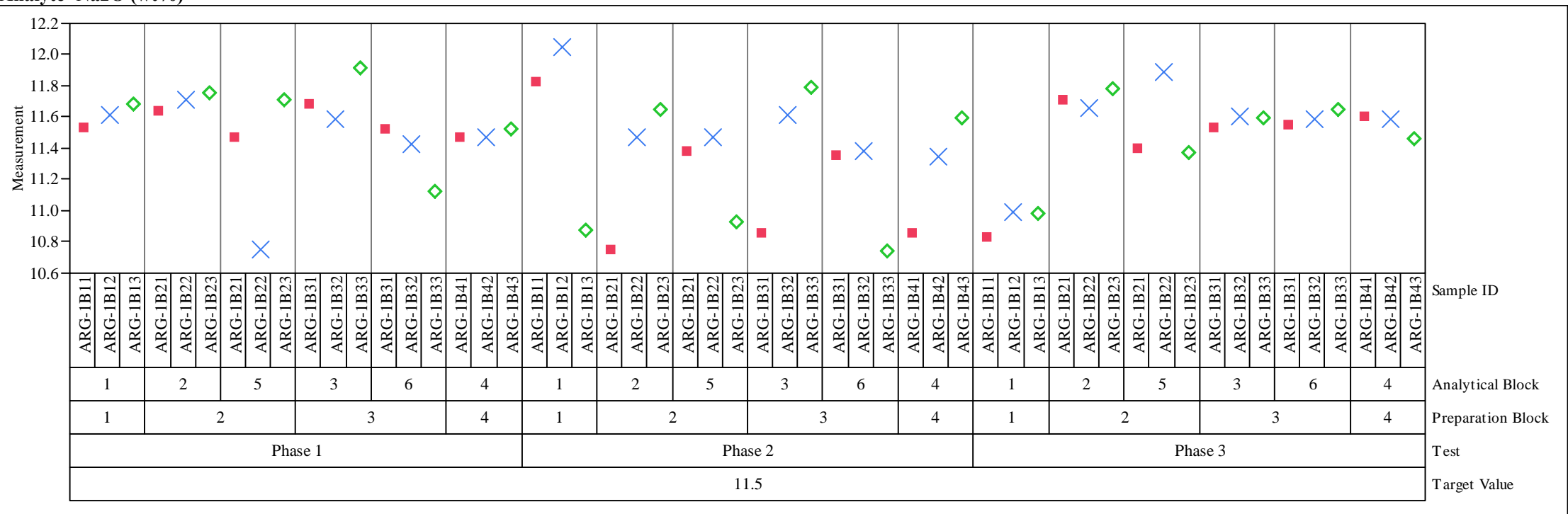


Exhibit A1. Variability Chart for ARG-1 Oxide Measurements

Analyte $=\mathrm{NiO}(\mathrm{wt} \%)$
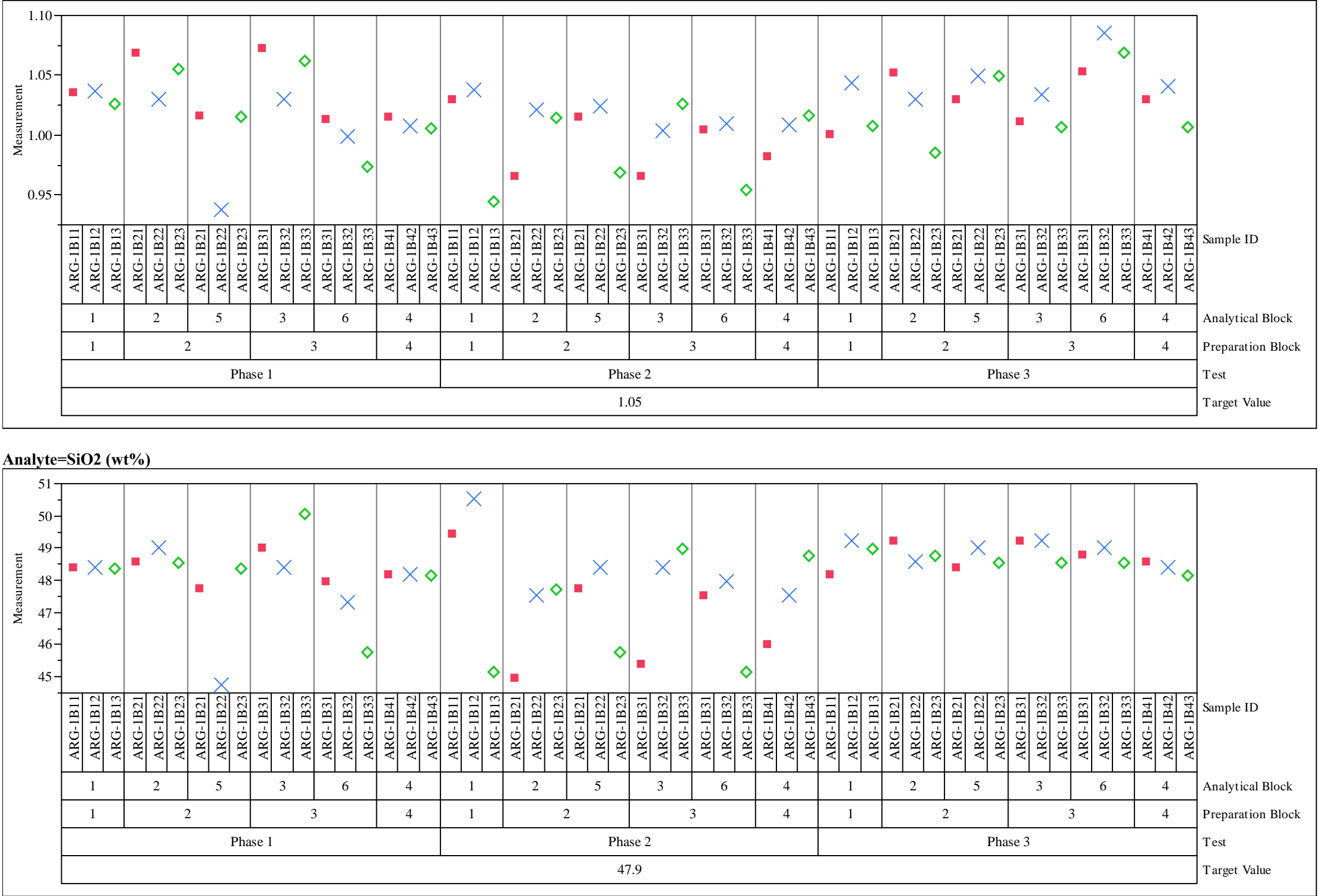
Exhibit A1. Variability Chart for ARG-1 Oxide Measurements

Analyte $=$ Sum of Oxides (wt\%)

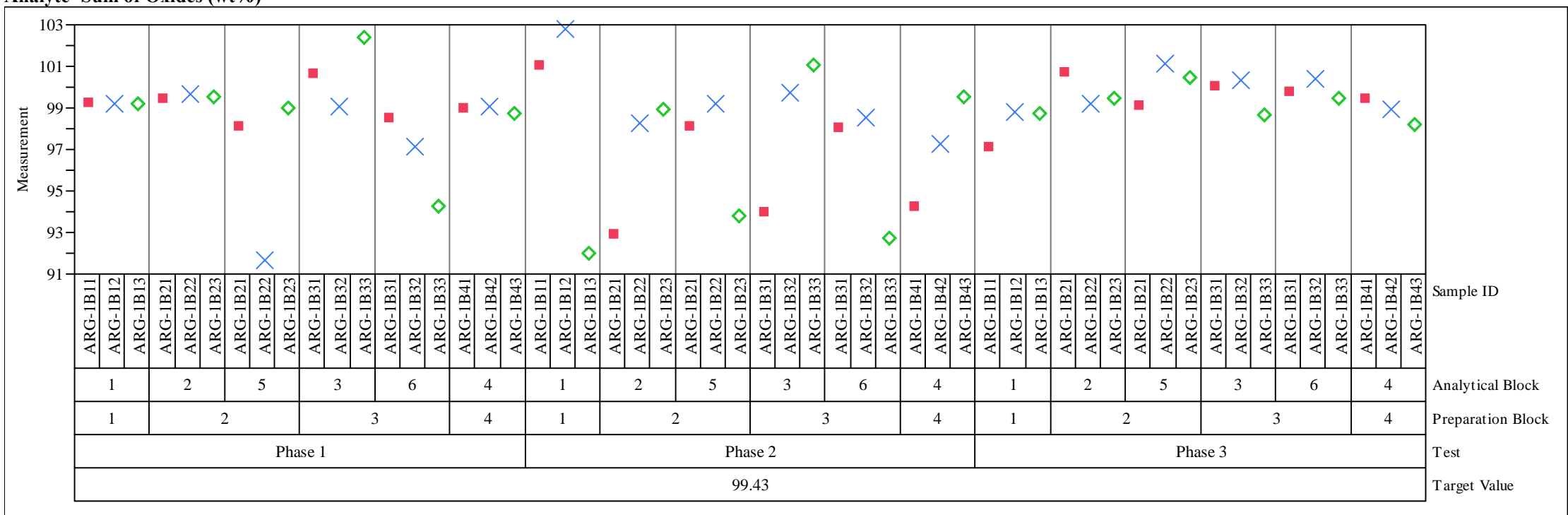

Analyte $=\mathrm{TiO} 2(\mathrm{wt} \%)$

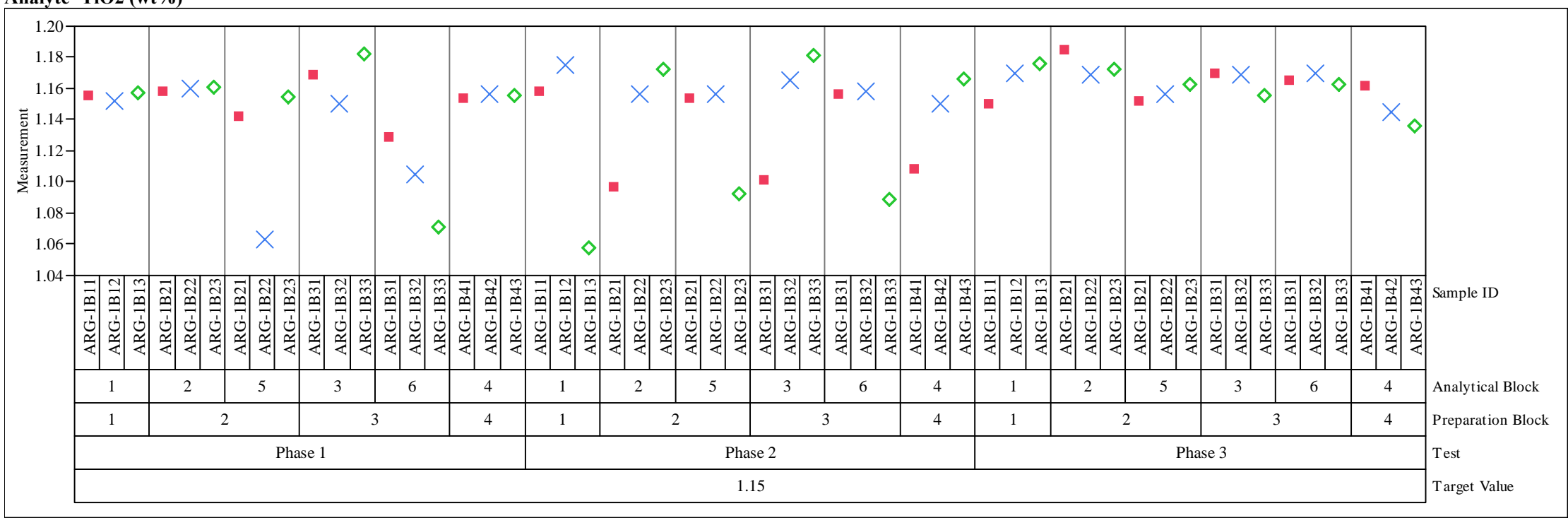


Exhibit A1. Variability Chart for ARG-1 Oxide Measurements

Analyte $=\mathrm{ZnO}(\mathrm{wt} \%)$

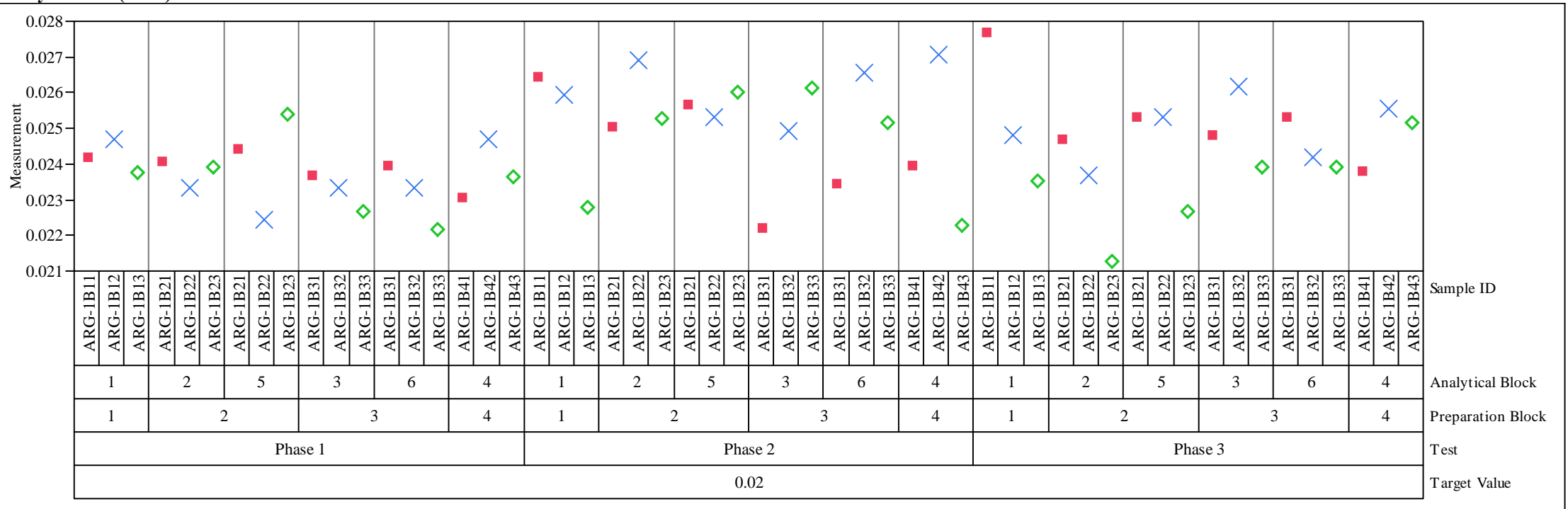

Analyte $=\mathrm{ZrO2}(\mathrm{wt} \%)$

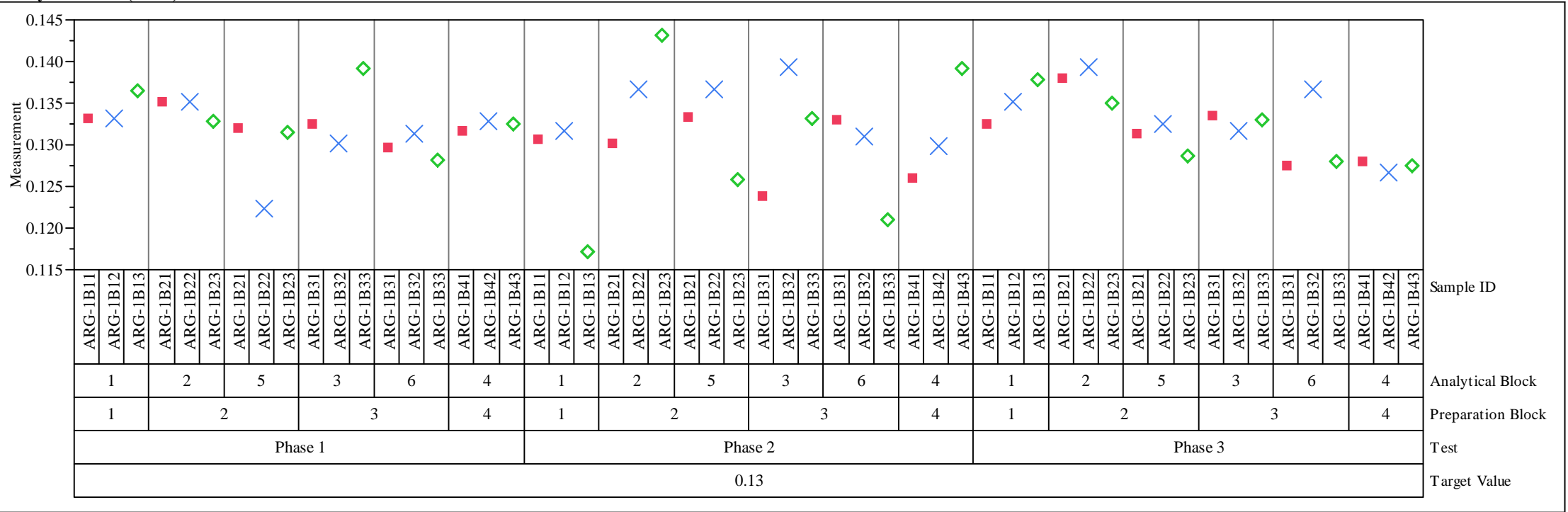




\section{Exhibit A2. Analysis of Variance to Estimate Preparation Block (PB) Versus Analytical Block (AB) Effects}

Response Measurement Analyte $=\mathrm{Al2O}(\mathrm{wt} \%)$, Target Value $=4.73$ Summary of Fit

\begin{tabular}{|c|c|c|c|c|}
\hline \multicolumn{3}{|c|}{ RSquare } & \multicolumn{2}{|l|}{0.476283} \\
\hline \multicolumn{3}{|c|}{ RSquare Adj } & \multicolumn{2}{|l|}{0.236246} \\
\hline \multicolumn{3}{|c|}{ Root Mean Square Error } & \multicolumn{2}{|l|}{$\begin{array}{l}0.236246 \\
0.117536\end{array}$} \\
\hline \multirow{2}{*}{\multicolumn{5}{|c|}{$\begin{array}{l}\text { Mean of Response } \\
\text { Observations (or Sum Wgts) }\end{array}$}} \\
\hline & & & & \\
\hline \multicolumn{5}{|c|}{ Analysis of Variance } \\
\hline Source & DF & Sum of Square & es Mean Square & F Ratio \\
\hline Model & 11 & 0.3015240 & 0.027411 & 1.9842 \\
\hline Error & 24 & 0.3315535 & 0.013815 & Prob $>$ F \\
\hline C. Total & 35 & 0.6330776 & & 0.0778 \\
\hline
\end{tabular}

The Mean Square per row by the Variance Component per column

\begin{tabular}{|c|c|c|}
\hline EMS & Intercept Ph: & ase-PB\&Random \\
\hline Intercept & 0 & 0 \\
\hline Phase-PB\&Random & 0 & 6 \\
\hline Phase-AB[Phase-PB]\&Random & 0 & 0 \\
\hline \multicolumn{3}{|c|}{ plus 1.0 times Residual Error Variance } \\
\hline \multicolumn{3}{|c|}{$\begin{array}{l}\text { Variance Component Estimates } \\
\text { Component }\end{array}$} \\
\hline Component & Var Comp Es & it Percent of Total \\
\hline Phase-PB\&Random & -0.00215 & -11.860 \\
\hline Phase-AB[Phase-PB]\&Random & $0.00648 \mathrm{~s}$ & 35.751 \\
\hline Residual & 0.013815 & 76.109 \\
\hline $\begin{array}{l}\text { Total } \\
\text { - }\end{array}$ & 0.01815 & 100. \\
\hline
\end{tabular}

These estimates based on equating Mean Squares to Expected Value.

Test Denominator Synthesis

MS Den DF Den Denom MS Synthesis

hase-PB\&Random $\quad 0.03328$

6 Phase-AB[Phase-PB]\&Random

Phase-AB[Phase-PB]\&Random 0.01381

24 Residual

Tests wrt Random Effects

SS MS Num DF Num F Ratio Prob $>$ F \begin{tabular}{llllll} 
Phase-PB\&Random & 0.10183 & 0.02037 & 5 & 0.6119 & 0.6968 \\
\hline
\end{tabular}

Phase-AB[Phase-PB]\&Random $0.19969 \quad 0.03328 \quad 6 \quad 6 \quad 2.4092 \quad 0.0577$

Response Measurement Analyte $=\mathbf{B 2 O 3}(\mathbf{w t} \%)$, Target Value $=8.67$

Summary of Fit

$\begin{array}{lr}\text { RSquare } & 0.553816 \\ \text { RSquare Adj } & 0.349314 \\ \text { Root Mean Square Error } & 0.262794 \\ \text { Mean of Response } & 7.968358 \\ \text { Observations (or Sum Wgts) } & 36\end{array}$

Mean of Response 7.968358

Analysis of Variance

Source DF Sum of Squares Mean Square F Ratio

Error $\quad 24-2.0572796$

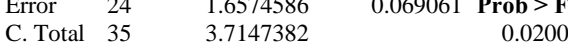

\section{Expected Mean Square}

The Mean Square per row by the Variance Component per column

EMS Intercept Phase-PB\&Random Phase-AB[Phase-PB]\&Random

Phase-PB\&Random

Phase-AB[Phase-PB]\&Random

plus 1.0 times Residual Error Variance

Variance Component Estimates

Component Var Comp Est Percent of Total

Phase-PB\&Random

$\begin{array}{lrr} & 0.004975 & 4.571 \\ & 0.034799 & 31.974\end{array}$

$\begin{array}{lll}\text { Phase-AB[Phase-PB]\&Random } & 0.0049951 & 31.974 \\ \text { Residual } & 0.069061 & 63.455\end{array}$

$\begin{array}{lrr}\text { Total } & 0.069835 & 63.455 \\ \end{array}$

These estimates based on equating Mean Squares to Expected Value.

Test Denominator Synthesis

Source MS Den DF Den Denom MS Synthesis

Phase-PB\&Random $\quad 0.17346 \quad 6$ Phase-AB[Phase-PB]\&Random

Phase-AB[Phase-PB]\&Random $0.06906 \quad 24$ Residual

\section{Tests wrt Random Effects}$$
4 \text { Residual }
$$

$\begin{array}{rrrrr}\text { SS } & \text { MS Num } & \text { DF Num F Ratio } & \text { Prob }>\text { F } \\ 1.01653 & 0.20331 & 5 & 1.1721 & 0.4192\end{array}$

Phase-PB\&Random

Phase-AB[Phase-PB]\&Random $\begin{array}{llllll}1.04075 & 0.17346 & 6 & 2.5117 & 0.0497\end{array}$ 


\section{Exhibit A2. Analysis of Variance to Estimate Preparation Block (PB) Versus Analytical Block (AB) Effects}

Response Measurement Analyte $=\mathrm{BaO}(\mathrm{wt} \%)$, Target Value $=0.09$ Summary of Fit

\begin{tabular}{|c|c|c|c|c|}
\hline \multirow{2}{*}{\multicolumn{3}{|c|}{ RSquare Adj }} & \multicolumn{2}{|l|}{0.483184} \\
\hline & & & 0.246309 & \\
\hline \multicolumn{3}{|c|}{ Root Mean Square Err } & 0.003393 & \\
\hline \multicolumn{3}{|c|}{ Mean of Response } & 0.092217 & \\
\hline \multicolumn{3}{|c|}{ Observations (or Sum Wgts) } & 36 & \\
\hline \multicolumn{5}{|c|}{ Analysis of Variance } \\
\hline Source & DF & Sum of Squares & s Mean Square & $\mathbf{F} \mathbf{R a}$ \\
\hline Model & 11 & 0.00025826 & 0.000023 & 2.0398 \\
\hline Error & 24 & 0.00027624 & 0.000012 & Prob $>$ F \\
\hline C. Total & 35 & 0.00053450 & & 0.0700 \\
\hline
\end{tabular}

The Mean Square per row by the Variance Component per column

\begin{tabular}{lcr} 
EMS & Intercept & Phase-PB\&Random P \\
Intercept & 0 & 0 \\
Phase-PB\&Random & 0 & 6 \\
Phase-AB[Phase-PB]\&Random & 0 & 0 \\
\multicolumn{3}{c}{} \\
plus 1.0 times Residual Error Variance \\
Variance Component Estimates \\
Component & Var Comp Est & Percent of Total \\
Phase-PB\&Random & $3.297 \mathrm{e}-6$ & 20.867 \\
Phase-AB[Phase-PB]\&Random & 0.000001 & 6.281 \\
Residual & $1.151 \mathrm{e}-5$ & 72.852 \\
Total & $1.58 \mathrm{e}-5$ & 100.000
\end{tabular}

These estimates based on equating Mean Squares to Expected Value.

Test Denominator Synthesis

MS Den DF Den Denom MS Synthesis

Phase-PB\&Random $\quad 1.45 e-5 \quad 6$ Phase-AB[Phase-PB]\&Random

Phase-AB[Phase-PB]\&Random $1.15 \mathrm{e}-5$

Tests wrt Random Effects

Source $\quad$ SS MS Num DF Num F Ratio Prob $>$ F

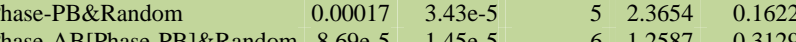

Response Measurement Analyte $=\mathrm{CaO}(\mathrm{wt} \%)$, Target Value $=1.43$

Summary of Fit

$\begin{array}{lr}\text { RSquare } & 0.55286 \\ \text { RSquare Adj } & 0.347921 \\ \text { Root Mean Square Error } & 0.037126 \\ \text { Mean of Response } & 1.445879 \\ \text { Observations (or Sum Wgts) } & 36\end{array}$

Mean of Response $\quad 1.445879$

Analysis of Variance

Source DF Sum of Squares Mean Square F Ratio

$\begin{array}{llll}\text { Error } & 24 & 0.03307963 & 0.001378 \quad \text { Prob }>\text { F }\end{array}$

$\begin{array}{lrrr}\text { C. Total } & 35 & 0.07398046 & 0.0204\end{array}$

\section{Expected Mean Square}

The Mean Square per row by the Variance Component per column

$\begin{array}{lccc}\text { EMS } & \text { Intercept } & \text { Phase-PB\&Random Phase-AB[Phase-PB]\&Random } \\ \text { Intercept } & 0 & 0 & 0\end{array}$

Phase-PB\&Random

plus 1.0 times Residual Error Variance

Variance Component Estimates

Component Var Comp Est Percent of Total

Vomp Est Percent of Total

$\begin{array}{lll}\text { Phase-PB\&Random } & 0.000457 & 20.759 \\ \text { Phase-AB[ } & 0.000365 & 16.585\end{array}$

Phase-AB[Phase-PB]\&Random

Total

0.0022

100.000

These estimates based on equating Mean Squares to Expected Value. Test Denominator Synthesis

Source MS Den DF Den Denom MS Synthesis

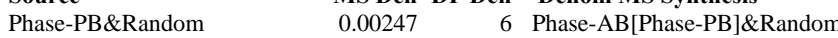

Phase-AB[Phase-PB]\&Random $0.00138 \quad 24$ Residual

\section{Tests wrt Random Effects}

\section{Source}

SS MS Num DF Num F Ratio Prob $>$ F

\begin{tabular}{llllll} 
Phase-PB\&Random & 0.02606 & 0.00521 & 5 & 2.1080 & 0.1952 \\
\hline
\end{tabular}

$\begin{array}{llllll}\text { Phase-AB[Phase-PB]\&Random } 0.01484 & 0.00247 & 6 & 1.7941 & 0.1429\end{array}$ 


\section{Exhibit A2. Analysis of Variance to Estimate Preparation Block (PB) Versus Analytical Block (AB) Effects}

Response Measurement Analyte $=\mathrm{Cr} 2 \mathrm{O} 3(\mathrm{wt} \%)$, Target Value $=0.09$ Summary of Fit

\begin{tabular}{|c|c|c|c|c|}
\hline \multicolumn{3}{|c|}{$\begin{array}{l}\text { RSquare } \\
\text { RSquare Adi }\end{array}$} & \multicolumn{2}{|l|}{0.642053} \\
\hline \multirow{2}{*}{\multicolumn{3}{|c|}{$\begin{array}{l}\text { RSquare Adj } \\
\text { Root Mean Square Error }\end{array}$}} & 0.477994 & \\
\hline & & & 0.004037 & \\
\hline \multicolumn{3}{|c|}{ Mean of Response } & 0.098276 & \\
\hline \multicolumn{3}{|c|}{ Observations (or Sum Wgts) } & 36 & \\
\hline \multicolumn{5}{|c|}{ Analysis of Variance } \\
\hline Source & DF & Sum of Square & s Mean Square & F Ratio \\
\hline Model & 11 & 0.00070166 & 0.000064 & 3.9135 \\
\hline Error & 24 & 0.00039118 & 0.000016 & Prob $>$ F \\
\hline C. Total & 35 & 0.0010928 & & 0.0025 \\
\hline
\end{tabular}

The Mean Square per row by the Variance Component per column

\begin{tabular}{|c|c|c|}
\hline EMS & Intercept Pha & se-PB\&Random \\
\hline Intercept & 0 & 0 \\
\hline Phase-PB\&Random & 0 & 6 \\
\hline Phase-AB[Phase-PB]\&Random & 0 & 0 \\
\hline \multicolumn{3}{|c|}{ plus 1.0 times Residual Error Variance } \\
\hline \multicolumn{3}{|c|}{ Variance Component Estimates } \\
\hline Component & Var Comp Est & Percent of Total \\
\hline Phase-PB\&Random & $9.312 \mathrm{e}-6$ & 28.241 \\
\hline Phase-AB[Phase-PB]\&Random & $7.364 \mathrm{e}-6$ & 22.331 \\
\hline Residual & $1.63 \mathrm{e}-5$ & 49.429 \\
\hline Total & 0.000033 & 100.000 \\
\hline
\end{tabular}

0.000033

100.000

These estimates based on equating Mean Squares to Expected Value. Test Denominator Synthesis

MS Den DF Den Denom MS Synthesis

Phase-PB\&Random $\quad 3.84 \mathrm{e}-5 \quad 6$ Phase-AB[Phase-PB]\&Random

Phase-AB[Phase-PB]\&Random $\quad 1.63 \mathrm{e}-5$

Tests wrt Random Effects

Source MS Num DF Num F Ratio Prob $>$ F

$\begin{array}{llllll}0.00047 & 9.43 e-5 & 5 & 2.4554 & 0.1523\end{array}$

Response Measurement Analyte $=\mathrm{CuO}(\mathbf{w t} \%)$, Target Value $=\mathbf{0}$

Summary of Fit

$\begin{array}{lr}\text { RSquare } & 0.931258 \\ \text { RSquare Adj } & 0.899751 \\ \text { Root Mean Square Error } & 0.002567 \\ \text { Mean of Response } & 0.009868 \\ \text { Observations (or Sum Wgts) } & 36\end{array}$

Mean of Response $\quad 0.009868$

Analysis of Variance

Source DF Sum of Squares Mean Square F Ratio

$\begin{array}{llll}11 & 0.00214201 & 0.000195 & 29.5574\end{array}$

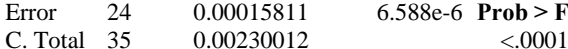

\section{Expected Mean Square}

The Mean Square per row by the Variance Component per column

EMS Intercept Phase-PB\&Random Phase-AB[Phase-PB]\&Random

$\begin{array}{rrr}0 & 0 & 0 \\ 0 & 6 & 3\end{array}$

Phase-PB\&Random

plus 1.0 times Residual Error Variance

Variance Component Estimates

Component Var Comp Est Percent of Total

Phase-PB\&Random $\quad 6.5840-5 \quad 87.456$

Phase-AB[Phase-PB]\&Random $\quad 2.856 \mathrm{e}-6 \quad 87.456$

\begin{tabular}{llr} 
Residual & $6.856 \mathrm{e}-6$ & 3.793 \\
\hline
\end{tabular}

$\begin{array}{lrr}\text { Total } & 6.588 \mathrm{e}-6 & \\ \text { Tesidual } & 7.529 \mathrm{e}-5 & 100.000\end{array}$

These estimates based on equating Mean Squares to Expected Value.

Test Denominator Synthesis

Source MS Den DF Den Denom MS Synthesis

Phase-PB\&Random $\quad 1.52 \mathrm{e}-5 \quad 6$ Phase-AB[Phase-PB]\&Random

Phase-AB[Phase-PB]\&Random $\quad 6.59 \mathrm{e}-6 \quad 24$ Residual

Tests wrt Random Effects

SS MS Num DF Num F Ratio Prob $>$ F

\begin{tabular}{|l|l|l|l|l|l}
0.00205 & 0.00041 & 5 & 27.0685 & 0.0005 \\
\hline
\end{tabular} 


\section{Exhibit A2. Analysis of Variance to Estimate Preparation Block (PB) Versus Analytical Block (AB) Effects}

Response Measurement Analyte=Fe2O3 (wt\%), Target Value=14 Summary of Fit

\begin{tabular}{|c|c|c|c|}
\hline Square & & 0.315326 & \\
\hline RSquare & Adj & 0.001517 & \\
\hline Root Me & an Square Error & 0.337976 & \\
\hline Mean of & Response & 13.88874 & \\
\hline Observat & ions (or Sum Wgts) & 36 & \\
\hline Analysis & of Variar & & \\
\hline Source & DF Sum of Squar & es Mean Square & \\
\hline Model & 1.26258 & 0.114780 & 1.0048 \\
\hline Error & 2.741465 & 0.114228 & Prob $>$ F \\
\hline C. Total & 4.00405 & & 0.4707 \\
\hline
\end{tabular}

The Mean Square per row by the Variance Component per column

\begin{tabular}{|c|c|c|}
\hline EMS & Intercept Pha: & e-PB\&Random \\
\hline Intercept & 0 & 0 \\
\hline Phase-PB\&Random & 0 & 6 \\
\hline Phase-AB[Phase-PB]\&Random & 0 & 0 \\
\hline \multicolumn{3}{|c|}{$\begin{array}{l}\text { plus } 1.0 \text { times Residual Error Variance } \\
\text { Variance Component Estimates }\end{array}$} \\
\hline \multicolumn{3}{|c|}{ Variance Component Estimates } \\
\hline Component & Var Comp Est & Percent of Total \\
\hline Phase-PB\&Random & 0.003134 & 2.732 \\
\hline Phase-AB[Phase-PB]\&Random & -0.00266 & -2.323 \\
\hline Residual & 0.114228 & 99.591 \\
\hline Total & 0.114697 & 100.000 \\
\hline
\end{tabular}

0.114697

These estimates based on equating Mean Squares to Expected Value.

Test Denominator Synthesis

MS Den DF Den Denom MS Synthesis

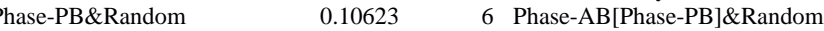

Phase-AB[Phase-PB]\&Random 0.11423

Tests wrt Random Effects

Source SS MS Num DF Num F Ratio Prob $>$ F

$\begin{array}{llllll} & 0.62518 & 0.12504 & 5 & 1.1770 & 0.4173\end{array}$

$\begin{array}{lllllll}\text { Phase-AB[Phase-PB]\&Random } & 0.6374 & 0.10623 & 6 & 0.9300 & 0.4915\end{array}$
Response Measurement Analyte $=\mathrm{K} 2 \mathrm{O}(\mathrm{wt} \%)$, Target Value $=\mathbf{2 . 7 1}$

Summary of Fit

$\begin{array}{lr}\text { RSquare } & 0.386106 \\ \text { RSquare Adj } & 0.104737 \\ \text { Root Mean Square Error } & 0.119957 \\ \text { Mean of Response } & 2.69295 \\ \text { Observations (or Sum Wgts) } & 36\end{array}$

Mean of Response 2.69295

Analysis of Variance

Source DF Sum of Squares Mean Square F Ratio

Error \begin{tabular}{llll}
24 & 0.21720773 & 0.019746 & 1.3722 \\
\hline
\end{tabular}

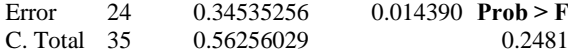

\section{Expected Mean Square}

The Mean Square per row by the Variance Component per column

$\begin{array}{lccc}\text { EMS } & \text { Intercept } & \text { Phase-PB\&Random Phase-AB[Phase-PB]\&Random } \\ \text { Intercept } & 0 & 0 & 0\end{array}$

Phase-PB\&Random

plus 1.0 times Residual Error Variance

Variance Component Estimates

Component Var Comp Est Percent of Total

Phase-PB\&Random

$\begin{array}{lrr}\text { Phase-PB\&Random } & 0.000974 & 5.988 \\ \text { Phase-AB } & 0.0009 & 5.535\end{array}$

$\begin{array}{lrr}\text { Phase-AB[Phase-PB]\&Rand } & 0.0039 & 5.535 \\ \text { Residual } & 0.01439 & 88.477\end{array}$

$\begin{array}{lrr}\text { Total } & 0.016264 & \\ \end{array}$

These estimates based on equating Mean Squares to Expected Value.

Test Denominator Synthesis

Source MS Den DF Den Denom MS Synthesis

Phase-PB\&Random $\quad 0.01709 \quad 6$ Phase-AB[Phase-PB]\&Rando

Phase-AB[Phase-PB]\&Random $0.01439 \quad 24$ Residual

\section{Tests wrt Random Effects}

Source SS MS Num DF Num F Ratio Prob $>$ F

$\begin{array}{llllll}\text { Phase-AB[Phase-PB]\&Random } 0.10254 & 0.02293 & & 5 & 1.3419 & 0.3611 \\ & 0.01709 & & 6 & 1.1877 & 0.3460\end{array}$ 


\section{Exhibit A2. Analysis of Variance to Estimate Preparation Block (PB) Versus Analytical Block (AB) Effects}

Response Measurement Analyte=Li2O (wt\%), Target Value=3.21 Summary of Fit

\begin{tabular}{|c|c|c|c|c|}
\hline quare & & & 0.501718 & \\
\hline RSquare & Adj & & 0.273339 & \\
\hline Root Me & an Squ & uare Error & 0.074837 & \\
\hline Mean of & Respo & onse & 3.164763 & \\
\hline Observat & ions ( & (or Sum Wgts) & 36 & \\
\hline Analysis & of $\mathrm{Va}$ & arian & & \\
\hline Source & DF & Sum of Squares & es Mean Square & \\
\hline Model & 11 & 0.13534137 & 0.012304 & 2.1969 \\
\hline Error & 24 & 0.13441437 & 0.005601 & Prob $>$ F \\
\hline C. Total & 35 & 0.26975574 & & 0.0519 \\
\hline
\end{tabular}

C. Total $35 \quad 0.2697557$

The Mean Square per row by the Variance Component per column

\begin{tabular}{|c|c|c|}
\hline EMS & Intercept Ph: & ase-PB\&Random \\
\hline Intercept & 0 & 0 \\
\hline Phase-PB\&Random & 0 & 6 \\
\hline Phase-AB[Phase-PB]\&Random & 0 & 0 \\
\hline \multicolumn{3}{|c|}{ plus 1.0 times Residual Error Variance } \\
\hline \multicolumn{3}{|c|}{ Variance Component Estimates } \\
\hline Component & Var Comp Es & t Percent of Total \\
\hline Phase-PB\&Random & -0.00152 & -19.772 \\
\hline Phase-AB[Phase-PB]\&Random & 0.003618 & 47.006 \\
\hline Residual & 0.005601 & 72.767 \\
\hline $\begin{array}{l}\text { Total } \\
\text { - }\end{array}$ & 0.007697 & 100. \\
\hline
\end{tabular}

These estimates based on equating Mean Squares to Expected Value.

Test Denominator Synthesis

MS Den DF Den Denom MS Synthesis

Phase-PB\&Random $\quad 0.01645$

6 Phase-AB[Phase-PB]\&Random

24 Residual

Tests wrt Random Effects

Source MS Num DF Num F Ratio Prob $>$ F

\begin{tabular}{lllllll}
\hline PB\&Random & 0.03662 & 0.00732 & 5 & 0.4451 & 0.8039
\end{tabular}

$\begin{array}{lllllll}\text { Phase-AB[Phase-PB]\&Random } & 0.09873 & 0.01645 & 6 & 2.9379 & 0.0271\end{array}$

Response Measurement Analyte $=\mathrm{MgO}(\mathrm{wt} \%)$, Target Value $=0.86$

Summary of Fit

$\begin{array}{lr}\text { RSquare } & 0.568216 \\ \text { RSquare Adj } & 0.370315 \\ \text { Root Mean Square Error } & 0.020047 \\ \text { Mean of Response } & 0.841495 \\ \text { Observations (or Sum Wgts) } & 36\end{array}$

Mean of Response $\quad 0.841495$

Analysis of Variance

Source DF Sum of Squares Mean Square F Ratio

Error $24-0.00964502$

C. Total $35-0.02233761-0.000402$ Prob $>$ F

\section{Expected Mean Square}

The Mean Square per row by the Variance Component per column

EMS Intercept Phase-PB\&Random Phase-AB[Phase-PB]\&Random

$\begin{array}{ccr}0 & 0 & 0 \\ 0 & 6 & 3\end{array}$

Phase-PB\&Random

plus 1.0 times Residual Error Variance

Variance Component Estimates

Component Var Comp Est Percent of Total

Phase-PB\&Random

Var Comp Est Percent of Tota

$\begin{array}{lll}\text { Phase-PB\&Random } & -0.00022 & -34.259 \\ \text { Phase-ABI } & 0.000448 & 70.755\end{array}$

$\begin{array}{lll}\text { Phase-AB[Phase-PB]\&Random } & 0.000448 & 70.755 \\ \text { Residual } & 0.000402 & 63.504\end{array}$

Total

0.000633

100.000

These estimates based on equating Mean Squares to Expected Value.

Test Denominator Synthesis

$\begin{array}{lrrr}\text { Source } & \text { MS Den } & \text { DF Den } & \text { Denom MS Synthesis } \\ \text { Phase-PB\&Random } & 0.00175 & 6 & \text { Phase-AB[Phase-PB]\&Random }\end{array}$

MS Den Den Denom MS Synthesis

Phase-AB[Phase-PB]\&Random $\quad 0.0004 \quad 24$ Residual

\section{Tests wrt Random Effects}

SS MS Num DF Num F Ratio Prob $>$ F

\begin{tabular}{lllll}
0.00222 & 0.00044 & 5 & 0.2546 & 0.9226 \\
\hline
\end{tabular} 


\section{Exhibit A2. Analysis of Variance to Estimate Preparation Block (PB) Versus Analytical Block (AB) Effects}

Response Measurement Analyte $=\mathrm{MnO}(\mathrm{wt} \%)$, Target Value $=1.89$ Summary of Fit

\begin{tabular}{|c|c|c|c|c|}
\hline \multirow{2}{*}{\multicolumn{3}{|c|}{ RSquare Adj }} & \multicolumn{2}{|l|}{0.584912} \\
\hline & & & 0.394663 & \\
\hline \multicolumn{3}{|c|}{ Root Mean Square Error } & 0.044103 & \\
\hline \multicolumn{3}{|c|}{ Mean of Response } & 1.828483 & \\
\hline \multicolumn{3}{|c|}{ Observations (or Sum Wgts) } & 36 & \\
\hline \multicolumn{5}{|c|}{ Analysis of Variance } \\
\hline Source & DF & Sum of Squares & s Mean Square & $\mathbf{F R a}$ \\
\hline Model & 11 & 0.06578020 & 0.005980 & 3.0745 \\
\hline Error & 24 & 0.04668153 & 0.001945 & Prob $>$ F \\
\hline C. Total & 35 & 0.11246173 & & 0.0104 \\
\hline
\end{tabular}

The Mean Square per row by the Variance Component per column

\begin{tabular}{|c|c|c|}
\hline EMS & Intercept Ph: & ase-PB\&Random \\
\hline Intercept & 0 & 0 \\
\hline Phase-PB\&Random & 0 & 6 \\
\hline Phase-AB[Phase-PB]\&Random & 0 & 0 \\
\hline \multicolumn{3}{|c|}{ plus 1.0 times Residual Error Variance } \\
\hline \multicolumn{3}{|c|}{ Variance Component Estimates } \\
\hline Component & Var Comp Es & t Percent of Total \\
\hline Phase-PB\&Random & -0.00122 & -38.390 \\
\hline Phase-AB[Phase-PB]\&Random & 0.00245 & 77.207 \\
\hline Residual & 0.00194 & 61.183 \\
\hline $\begin{array}{l}\text { Total } \\
\text { - }\end{array}$ & $0.00317 \mathrm{~s}$ & 100. \\
\hline
\end{tabular}

These estimates based on equating Mean Squares to Expected Value.

Test Denominator Synthesis

MS Den DF Den Denom MS Synthesis

Phase-PB\&Random $\quad 0.00931 \quad 6$ Phase-AB[Phase-PB]\&Random

Phase-AB[Phase-PB]\&Random 0.00195

Tests wrt Random Effects

Source SS MS Num DF Num F Ratio Prob $>$ F

$\begin{array}{llllll} & 0.00993 & 0.00199 & 5 & 0.2133 & 0.9444\end{array}$

Phase-AB[Phase-PB]\&Random $\begin{array}{lllllll}0.05585 & 0.00931 & 6 & 4.7857 & 0.002\end{array}$

Response Measurement Analyte $=\mathrm{Na2O}(\mathrm{wt} \%)$, Target Value $=11.5$

Summary of Fit

$\begin{array}{lr}\text { RSquare } & 0.366715 \\ \text { RSquare Adj } & 0.07646 \\ \text { Root Mean Square Error } & 0.303192 \\ \text { Mean of Response } & 11.46699 \\ \text { Observations (or Sum Wgts) } & 36\end{array}$

Mean of Response 11.46699

Analysis of Variance

Source DF Sum of Squares Mean Square F Ratio

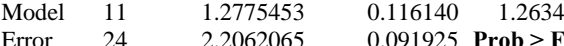

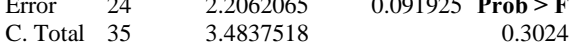

\section{Expected Mean Squares}

The Mean Square per row by the Variance Component per column

EMS Intercept Phase-PB\&Random Phase-AB[Phase-PB]\&Random

Phase-PB\&Random

Phase-AB[Phase-PB]\&Random

plus 1.0 times Residual Error Variance

Variance Component Estimates

Component Var Comp Est Percent of Total

Phase-PB\&Random

Var Comp Est Percent of Total

Phase-PB\&Random $\quad 0.007106 \quad 7.060$

Phase-AB[Phase-PB]\&Random

Total

0.100643

100.000

These estimates based on equating Mean Squares to Expected Value. Test Denominator Synthesis

Source MS Den DF Den Denom MS Synthesis

Phase-PB\&Random $\quad 0.09676 \quad 6$ Phase-AB[Phase-PB]\&Random

Phase-AB[Phase-PB]\&Random $0.09193 \quad 24$ Residual

\section{Tests wrt Random Effects}

Source SS MS Num DF Num F Ratio Prob $>$ F

\begin{tabular}{llllll} 
Phase-AB[Phase-PB]\&Random 0.58056 & 0.09676 & & 5 & 1.4406 & 0.3318 \\
\hline & 0.0526 & & & & 0.4173
\end{tabular} 


\section{Exhibit A2. Analysis of Variance to Estimate Preparation Block (PB) Versus Analytical Block (AB) Effects}

Response Measurement Analyte=NiO (wt\%), Target Value $=1.05$ Summary of Fit

\begin{tabular}{|c|c|c|c|c|}
\hline \multicolumn{5}{|c|}{0.60098} \\
\hline \multicolumn{3}{|c|}{ RSquare Adj } & 0.418095 & \\
\hline \multicolumn{3}{|c|}{ Root Mean Square Error } & 0.026981 & \\
\hline \multicolumn{3}{|c|}{ Mean of Response } & 1.018919 & \\
\hline \multicolumn{5}{|c|}{ Observations (or Sum Wgts) } \\
\hline \multicolumn{5}{|c|}{ Analysis of Variance } \\
\hline Source & DF & Sum of Squares & s Mean Square & \\
\hline Model & 11 & 0.02631489 & 0.002392 & 3.2861 \\
\hline Error & 24 & 0.01747177 & 0.000728 & Prob $>$ F \\
\hline C. Total & 35 & 0.04378667 & & 0.0072 \\
\hline
\end{tabular}

The Mean Square per row by the Variance Component per column

\begin{tabular}{lcr} 
EMS & Intercept & Phase-PB\&Random P \\
Intercept & 0 & 0 \\
Phase-PB\&Random & 0 & 6 \\
Phase-AB[Phase-PB]\&Random & 0 & 0 \\
\multicolumn{3}{c}{} \\
plus 1.0 times Residual Error Variance \\
Variance Component Estimates \\
Component & Var Comp Est & Percent of Total \\
Phase-PB\&Random & -0.00009 & -7.026 \\
Phase-AB[Phase-PB]\&Random & 0.000636 & 49.911 \\
Residual & 0.000728 & 57.115 \\
Total & 0.001275 & 100.000
\end{tabular}

These estimates based on equating Mean Squares to Expected Value.

Test Denominator Synthesis

MS Den DF Den Denom MS Synthesis

Phase-PB\&Random $\quad 0.00264 \quad 6$ Phase-AB[Phase-PB]\&Random

Phase-AB[Phase-PB]\&Random 0.00073

Tests wrt Random Effects

$\begin{array}{lll}\text { Source } & \text { SS MS Num DF Num F Ratio Prob }>\text { F }\end{array}$

$\begin{array}{llllll}0.0105 & 0.0021 & 5 & 0.7962 & 0.5897\end{array}$

Phase-AB[Phase-PB]\&Random $\begin{array}{llllll}0.01582 & 0.00264 & 6 & 3.6216 & 0.0106\end{array}$

Response Measurement Analyte $=\mathrm{SiO} 2(\mathrm{wt} \%)$, Target Value $=47.9$

Summary of Fit

$\begin{array}{lr}\text { RSquare } & 0.486224 \\ \text { RSquare Adj } & 0.250743 \\ \text { Root Mean Square Error } & 1.167939 \\ \text { Mean of Response } & 47.95003 \\ \text { Observations (or Sum Wgts) } & 36\end{array}$

Mean of Response 47.95003

Analysis of Variance

Source DF Sum of Squares Mean Square F Ratio

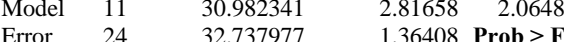

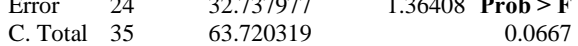

\section{Expected Mean Square}

The Mean Square per row by the Variance Component per column

$\begin{array}{lccc}\text { EMS } & \text { Intercept } & \text { Phase-PB\&Random Phase-AB[Phase-PB]\&Random } \\ \text { Intercept } & 0 & 0 & 0\end{array}$

Phase-PB\&Random

plus 1.0 times Residual Error Variance

Variance Component Estimates

Component Var Comp Est Percent of Total

Vomp Est Percent of Total

$\begin{array}{lll}\text { Phase-PB\&Random } & 0.237051 & 12.678 \\ \text { Phase-AB[Pase } & 0.268664 & 14.369\end{array}$

Phase-AB[Phase-PB]\&Random

Total

1.869797

100.000

These estimates based on equating Mean Squares to Expected Value.

Test Denominator Synthesis

MS Den DF Den Denom MS Synthesis

Phase-PB\&Random $2.17007 \quad 6$ Phase-AB[Phase-PB]\&Random

Phase-AB[Phase-PB]\&Random $1.36408 \quad 24$ Residual

Tests wrt Random Effects

SS MS Num DF Num F Ratio Prob $>$ F

\begin{tabular}{lllll}
17.9619 & 3.59238 & 5 & 1.6554 & 0.2775 \\
\hline
\end{tabular} 


\section{Exhibit A2. Analysis of Variance to Estimate Preparation Block (PB) Versus Analytical Block (AB) Effects}

Response Measurement Analyte=Sum of Oxides (wt\%), Target Value=99.43 Summary of Fit

\begin{tabular}{|c|c|c|c|c|}
\hline \multicolumn{3}{|c|}{ RSquare } & \multicolumn{2}{|l|}{0.407881} \\
\hline \multicolumn{3}{|c|}{ RSquare Adj } & 0.136494 & \\
\hline \multicolumn{3}{|c|}{ Root Mean Square Error } & 2.407604 & \\
\hline \multicolumn{3}{|c|}{ Mean of Response } & 98.39457 & \\
\hline \multicolumn{3}{|c|}{ Observations (or Sum Wgts) } & 36 & \\
\hline \multicolumn{5}{|c|}{ Analysis of Variance } \\
\hline Source & DF & Sum of Square & es Mean Square & $\mathbf{F R}$ \\
\hline Model & 11 & $95.8310^{3}-10$ & 8.71191 & 1.5029 \\
\hline Error & 24 & 139.1173 & 5.79655 & Prob $>$ F \\
\hline C. Tota & 35 & 234.9483 & & 0.1946 \\
\hline
\end{tabular}

Expected Mean Square

The Mean Square per row by the Variance Component per column

\begin{tabular}{lcr} 
EMS & Intercept & Phase-PB\&Random \\
Intercept & 0 & 0 \\
Phase-PB\&Random & 0 & 6 \\
Phase-AB[Phase-PB]\&Random & 0 & 0 \\
\multicolumn{3}{c}{} \\
plus 1.0 times Residual Error Variance \\
Variance Component Estimates \\
Component & Var Comp Est & Percent of Total \\
Phase-PB\&Random & 0.378185 & 5.559 \\
Phase-AB[Phase-PB]\&Random & 0.627981 & 9.231 \\
Residual & 5.796555 & 85.209 \\
Total & 6.802721 & 100.000
\end{tabular}

These estimates based on equating Mean Squares to Expected Value. Test Denominator Synthesis

MS Den DF Den Denom MS Synthesis

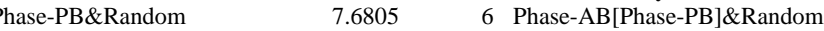

Phase-AB[Phase-PB]\&Random 5.79655

Tests wrt Random Effects

SS MS Num DF Num F Ratio Prob $>$ F

\begin{tabular}{|l|l|l|l|l|l} 
Phase-PB\&Random & 49.748 & 9.94961 & 5 & 1.2954 & 0.3760 \\
\hline
\end{tabular}

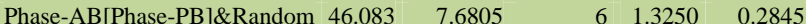

Response Measurement Analyte $=\mathrm{TiO} 2$ (wt\%), Target Value $=1.15$

Summary of Fit

$\begin{array}{lr}\text { RSquare } & 0.444705 \\ \text { RSquare Adj } & 0.190195 \\ \text { Root Mean Square Error } & 0.028767 \\ \text { Mean of Response } & 1.147074 \\ \text { Observations (or Sum Wgts) } & 36\end{array}$

Mean of Response $\quad 1.147074$

Analysis of Variance

Source DF Sum of Squares Mean Square F Ratio

Error $24 \quad 0.01986137$

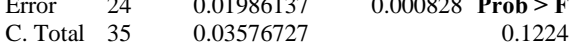

\section{Expected Mean Square}

The Mean Square per row by the Variance Component per column

$\begin{array}{lccc}\text { EMS } & \text { Intercept } & \text { Phase-PB\&Random Phase-AB[Phase-PB]\&Random } \\ \text { Intercept } & 0 & 0 & 0\end{array}$

Phase-PB\&Random

plus 1.0 times Residual Error Variance

Variance Component Estimates

Component Var Comp Est Percent of Total

Phase-PB\&Randor

$\begin{array}{lrr}\text { Phase-PB\&Random } & -6.7 \mathrm{e}-5 & -6.516 \\ & 0.000267 & 25.984\end{array}$

$\begin{array}{lll}\text { Phase-AB[Phase-PB]\&Random } & 0.000267 & 25.984 \\ \text { Residual } & 0.000828 & 80.532\end{array}$

$\begin{array}{lrr}\text { Total } & 0.000828 & 80.532 \\ & 0.001028 & 100.000\end{array}$

These estimates based on equating Mean Squares to Expected Value.

Test Denominator Synthesis

Source MS Den DF Den Denom MS Synthesis

Phase-PB\&Random $\quad 0.00163 \quad 6$ Phase-AB[Phase-PB]\&Rando

Phase-AB[Phase-PB]\&Random $0.00083 \quad 24$ Residual

\section{Tests wrt Random Effects}

SS MS Num DF Num F Ratio Prob $>$ F

\begin{tabular}{llllll} 
Phase-PB\&Random & 0.00613 & 0.00123 & 5 & 0.7533 & 0.6133 \\
\hline
\end{tabular}

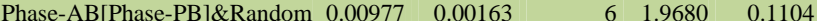




\section{Exhibit A2. Analysis of Variance to Estimate Preparation Block (PB) Versus Analytical Block (AB) Effects}

Response Measurement Analyte $=\mathrm{ZnO}(\mathrm{wt} \%)$, Target Value $=0.02$ Summary of Fit

\begin{tabular}{|c|c|c|c|c|}
\hline \multirow{2}{*}{\multicolumn{3}{|c|}{$\begin{array}{l}\text { RSquare } \\
\text { RSquare Adj }\end{array}$}} & \multicolumn{2}{|l|}{0.424851} \\
\hline & Adj & & 0.161241 & \\
\hline \multicolumn{3}{|c|}{ Root Mean Square Error } & 0.001236 & \\
\hline \multicolumn{3}{|c|}{ Mean of Response } & 0.024322 & \\
\hline \multicolumn{3}{|c|}{ Observations (or Sum Wgts) } & 36 & \\
\hline \multicolumn{5}{|c|}{ Analysis of Variance } \\
\hline Source & DF & Sum of Square & es Mean Square & \\
\hline Model & 11 & 0.0000270 & $2.4612 \mathrm{e}-6$ & 1.6117 \\
\hline Error & 24 & 0.0000366 & $1.5271 \mathrm{e}-6$ & Prob $>F$ \\
\hline C. Tota & 35 & 0.0000637 & & 0.1585 \\
\hline
\end{tabular}

The Mean Square per row by the Variance Component per column

\begin{tabular}{|c|c|c|}
\hline EMS & Intercept Pha: & e-PB\&Random \\
\hline Intercept & 0 & 0 \\
\hline Phase-PB\&Random & 0 & 6 \\
\hline Phase-AB[Phase-PB]\&Random & 0 & 0 \\
\hline \multicolumn{3}{|c|}{$\begin{array}{l}\text { plus } 1.0 \text { times Residual Error Variance } \\
\text { Variance Component Estimates }\end{array}$} \\
\hline \multicolumn{3}{|c|}{ Variance Component Estimates } \\
\hline Component & Var Comp Est & Percent of Total \\
\hline Phase-PB\&Random & $7.001 \mathrm{e}-7$ & 36.807 \\
\hline Phase-AB[Phase-PB]\&Random & $-3.25 e-7$ & -17.092 \\
\hline Residual & $1.527 \mathrm{e}-6$ & \\
\hline Total & $1.902 \mathrm{e}-6$ & 100.000 \\
\hline
\end{tabular}

These estimates based on equating Mean Squares to Expected Value.

Test Denominator Synthesis

MS Den DF Den Denom MS Synthesis

$\begin{array}{llll}\text { Phase-PB\&Random } & 5.52 \mathrm{e}-7 & 6 & \text { Phase-AB[Phase-PB]\&Random }\end{array}$

Phase-AB[Phase-PB]\&Random $1.53 \mathrm{e}-6$

Tests wrt Random Effects

SS MS Num DF Num F Ratio Prob $>$ F

\begin{tabular}{lllll|l|l|}
\hline Phase-PB\&Random & $2.38 \mathrm{e}-5$ & $4.75 \mathrm{e}-6$ & 5 & 8.6128 & 0.0104 \\
\hline
\end{tabular}

Phase-AB[Phase-PB]\&Random $3.31 \mathrm{e}-6 \quad 5.52 \mathrm{e}-7 \quad 6 \quad 6 \quad 0.3613 \quad 0.8961$

Response Measurement Analyte $=\mathbf{Z r O 2}(\mathrm{wt} \%)$, Target Value $=\mathbf{0 . 1 3}$

Summary of Fit

$\begin{array}{lr}\text { RSquare } & 0.345542 \\ \text { RSquare Adj } & 0.045582 \\ \text { Root Mean Square Error } & 0.004694 \\ \text { Mean of Response } & 0.13218 \\ \text { Observations (or Sum Wgts) } & 36\end{array}$

Analysis of Variance

Source DF Sum of Squares Mean Square F Ratio

$\begin{array}{lll}0.00027920 & 0.000025 & 1.1520\end{array}$

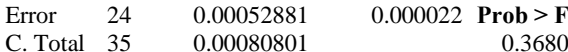

\section{Expected Mean Square}

The Mean Square per row by the Variance Component per column

EMS $\quad$ Intercept Phase-PB\&Random Phase-AB[Phase-PB]\&Random

$\begin{array}{rrr}0 & 0 & 0 \\ 0 & 6 & 3 \\ 0 & 0 & 3\end{array}$

Phase-AB[Phase-PB]\&Random

plus 1.0 times Residual Error Variance

Variance Component Estimates

Component Var Comp Est Percent of Total

ar Comp Est Percent of Tota

$\begin{array}{lll}\text { Phase-PB\&Random } & -3.13 \mathrm{e}-6 & -13.701 \\ & 3.964 \mathrm{e}-6 & 17.337\end{array}$

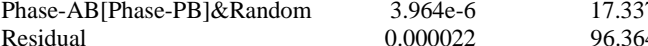

Total

$2.287 \mathrm{e}-5$

100.000

These estimates based on equating Mean Squares to Expected Value.

Test Denominator Synthesis

Source MS Den DF Den Denom MS Synthesis

Phase-PB\&Random $\quad 3.39$ e- $5 \quad 6$ Phase-AB[Phase-PB]\&Random

Phase-AB[Phase-PB]\&Random $\quad 2.2 \mathrm{e}-5 \quad 24$ Residual

\section{Tests wrt Random Effects}

SS MS Num DF Num F Ratio Prob $>$ F

\begin{tabular}{rr|rrrr}
$7.56 \mathrm{e}-5$ & $1.51 \mathrm{e}-5$ & 5 & 0.4460 & 0.8033 \\
\hline
\end{tabular} 


\section{Exhibit A3. Analysis of Variance to Estimate Analytical Block (AB) Effects}

\begin{tabular}{|c|c|}
\hline $\begin{array}{l}\text { Response Measurement Type of Material=ARG-1, Analyte=Al2O3 (wt\%), } \\
\text { Target Value }=4.73 \\
\text { Summary of Fit }\end{array}$ & $\begin{array}{l}\text { Response Measurement Type of Material=ARG-1, Analyte=BaO (wt } \%) \text {, } \\
\text { Target Value }=0.09 \\
\text { Summary of Fit }\end{array}$ \\
\hline 0.432173 & 0.479088 \\
\hline RSquare Adj & RSquare Adj \\
\hline Root Mean Square Error & Root Mean Square Error \\
\hline Mean of Response & Mean of Response \\
\hline Observations (or Sum Wgts) 54 & Observations (or Sum Wgts) 54 \\
\hline Analysis of Variance & Analysis of Variance \\
\hline Source DF Sum of Squares Mean Square F Ratio & Source DF Sum of Squares Mean Square F Ratio \\
\hline $\begin{array}{lllll}\text { Model } & 17 & 0.38350008 & 0.022559 & 1.6117\end{array}$ & $\begin{array}{llll}\text { Model } & 17 & 0.00036496 & 0.000021\end{array}$ \\
\hline 0.013997 Prob $>$ F & 0.000011 Prob $>$ F \\
\hline C. Total 53 & C. Total 53 \\
\hline Expected Mean Squares & Expected Mean Squares \\
\hline The Mean Square per row by the Variance Component per column & The Mean Square per row by the Variance Component per column \\
\hline Intercept Phase-AB\&Random & Intercept Phase-AB\&Random \\
\hline $\begin{array}{ll}0 & 0\end{array}$ & $\begin{array}{ll}0 & 0\end{array}$ \\
\hline Phase-AB\&Random & Phase-AB\&Random \\
\hline plus 1.0 times Residual Error Variance & plus 1.0 times Residual Error Variance \\
\hline Variance Component Estimates & Variance Component Estimates \\
\hline Component $\quad$ Var Comp Est Percent of Total & Component $\quad$ Var Comp Est Percent of Total \\
\hline Phase-AB\&Random $\quad 0.002854 \quad 16.938$ & Phase-AB\&Random $\quad 3.482 \mathrm{e}-6 \quad 24.005$ \\
\hline Residual & Residual \\
\hline 0.016851 & $1.45 \mathrm{e}-5$ \\
\hline $\begin{array}{l}\text { These estimates based on equating Mean Squares to Expected Value. } \\
\text { Test Denominator Synthesis }\end{array}$ & $\begin{array}{l}\text { These estimates based on equating Mean Squares to Expected Value. } \\
\text { Test Denominator Synthesis }\end{array}$ \\
\hline Source $\quad$ MS Den DF Den Denom MS Synthesis & Source $\quad$ MS Den DF Den Denom MS Synthesis \\
\hline Phase-AB\&Random $\quad 0.014 \quad 36$ Residual & Phase-AB\&Random $\quad 1.1 \mathrm{e}-5 \quad 36$ Residual \\
\hline Tests wrt Random Effects & Tests wrt Random Effects \\
\hline $\begin{array}{lrrrrr}\text { Source } & \text { SS } & \text { MS Num } & \text { DF Num F Ratio } & \text { Prob }>\text { F } \\
\text { Phase-AB\&Random } & 0.3835 & 0.02256 & 17 & 1.6117 & 0.1125\end{array}$ & \begin{tabular}{lrrrrr|} 
Source & SS & MS Num & DF Num & F Ratio & Prob > F \\
Phase-AB\&Random & 0.00036 & $2.15 \mathrm{e}-5$ & 17 & 1.9476 & 0.0458
\end{tabular} \\
\hline $\begin{array}{l}\text { Response Measurement Type of Material=ARG-1, Analyte=B2O3 (wt\%), } \\
\text { Target Value }=8.67 \\
\text { Summary of Fit }\end{array}$ & $\begin{array}{l}\text { Response Measurement Type of Material=ARG-1, Analyte=CaO }(w t \%) \text {, } \\
\text { Target Value }=1.43 \\
\text { Summary of Fit }\end{array}$ \\
\hline 0.509485 & 0.493477 \\
\hline RSquare Adj & RSquare Adj \\
\hline Root Mean Square Error & Root Mean Square Error \\
\hline Mean of Response & Mean of Response \\
\hline Observations (or Sum Wgts) $\quad 54$ & Observations (or Sum Wgts) $\quad 54$ \\
\hline Analysis of Variance & Analysis of Variance \\
\hline Source DF Sum of Squares Mean Square F Ratio & Source DF Sum of Squares Mean Square F Ratio \\
\hline $\begin{array}{lllll}\text { Model } & 17 & 2.5765986 & 0.151565 & 2.1995\end{array}$ & $\begin{array}{lllll}\text { Model } & 17 & 0.04556753 & 0.002680 & 2.0631\end{array}$ \\
\hline 0.068907 Prob $>$ F & 0.001299 Prob $>$ F \\
\hline C. Total 53 & C. Total 53 \\
\hline Expected Mean Squares & Expected Mean Squares \\
\hline The Mean Square per row by the Variance Component per column & The Mean Square per row by the Variance Component per column \\
\hline Intercept Phase-AB\&Random & Intercept Phase-AB\&Random \\
\hline $0 \quad 0$ & 0 \\
\hline Phase-AB\&Random & Phase-AB\&Random \\
\hline plus 1.0 times Residual Error Variance & plus 1.0 times Residual Error Variance \\
\hline Variance Component Estimates & Variance Component Estimates \\
\hline Component Var Comp Est Percent of Total & Component Var Comp Est Percent of Total \\
\hline Phase-AB\&Random $\quad 0.027552 \quad 28.564$ & Phase-AB\&Random $\quad 0.00046 \quad 26.165$ \\
\hline Residual & Residual \\
\hline 0.09646 & 100.000 \\
\hline $\begin{array}{l}\text { These estimates based on equating Mean Squares to Expected Value. } \\
\text { Test Denominator Synthesis }\end{array}$ & $\begin{array}{l}\text { These estimates based on equating Mean Squares to Expected Value. } \\
\text { Test Denominator Synthesis }\end{array}$ \\
\hline $\begin{array}{lll}\text { Source } & \text { MS Den DF Den Denom MS Synthesis }\end{array}$ & $\begin{array}{ll}\text { Source } & \text { MS Den DF Den Denom MS Synthesis }\end{array}$ \\
\hline Phase-AB\&Random $0.06891 \quad 36$ Residual & Phase-AB\&Random $\quad 0.0013 \quad 36$ Residual \\
\hline Tests wrt Random Effects & Tests wrt Random Effects \\
\hline Source $\quad$ SS MS Num DF Num F Ratio Prob $>$ F & Source $\quad$ SS MS Num DF Num F Ratio Prob $>$ F \\
\hline 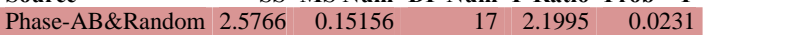 & \begin{tabular}{|l|l|l|l|l|l} 
Phase-AB\&Random & 0.04557 & 0.00268 & 17 & 2.0631 & 0.0335
\end{tabular} \\
\hline
\end{tabular}




\section{Exhibit A3. Analysis of Variance to Estimate Analytical Block (AB) Effects}

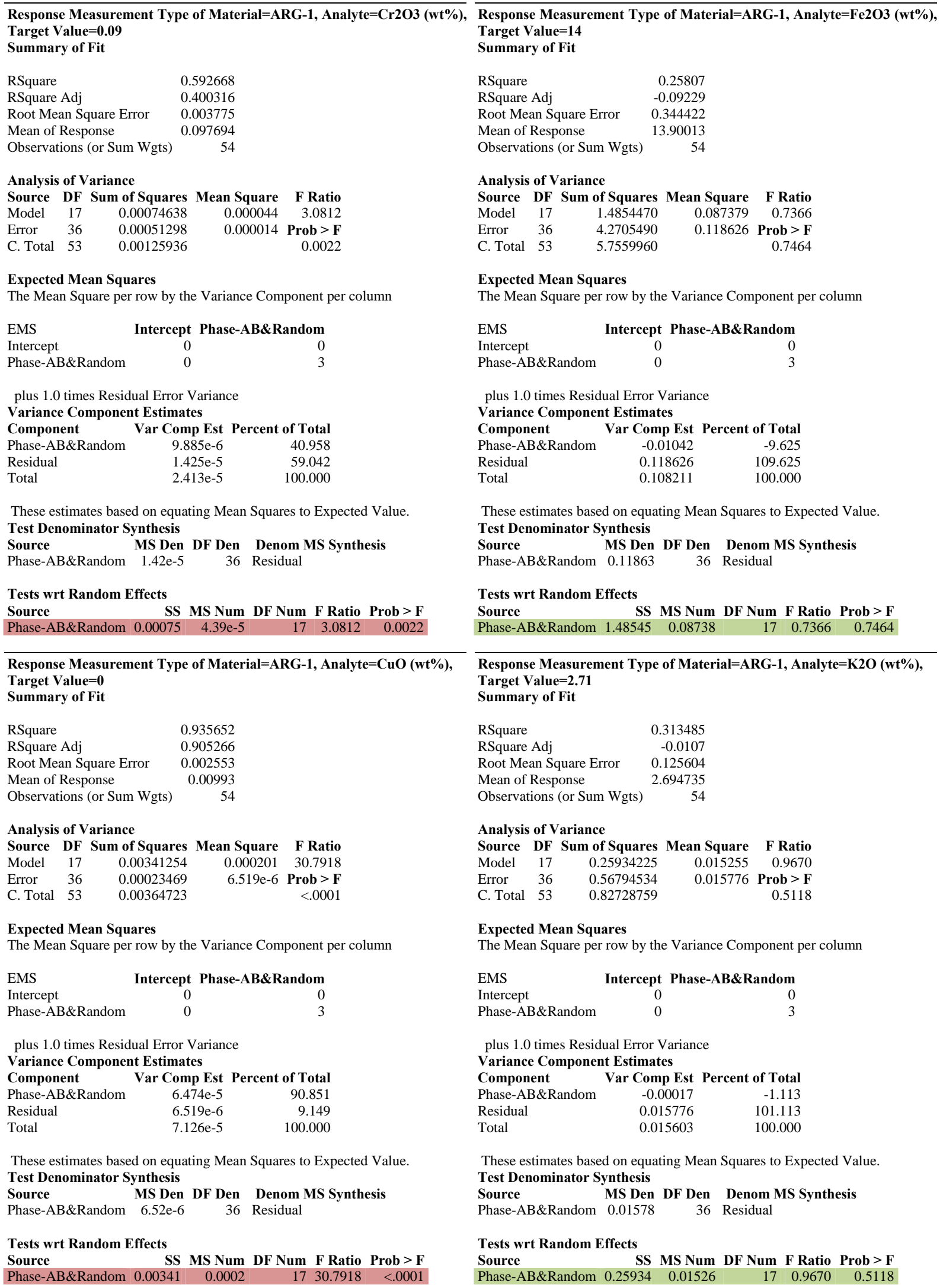




\section{Exhibit A3. Analysis of Variance to Estimate Analytical Block (AB) Effects}

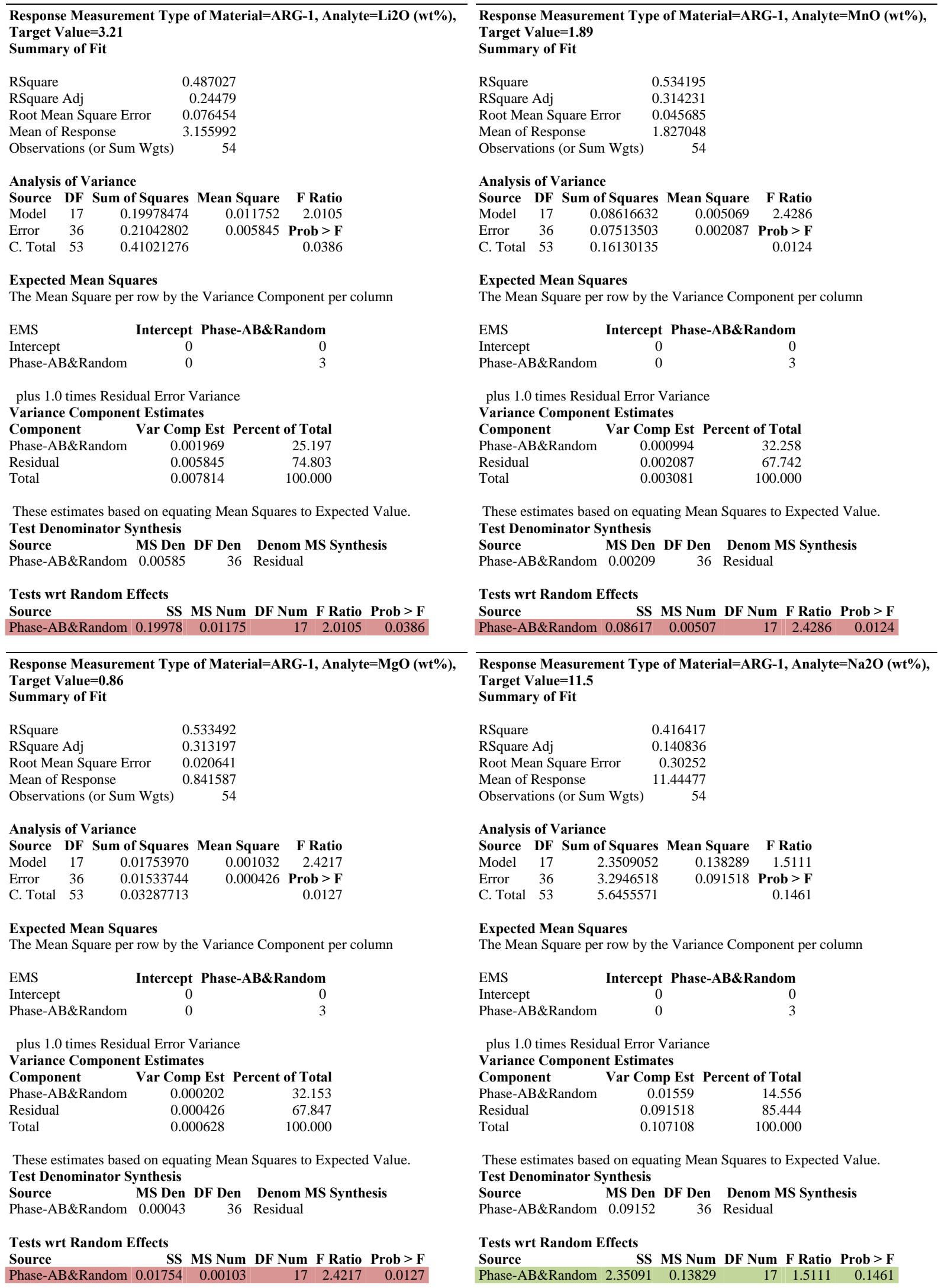




\section{Exhibit A3. Analysis of Variance to Estimate Analytical Block (AB) Effects}

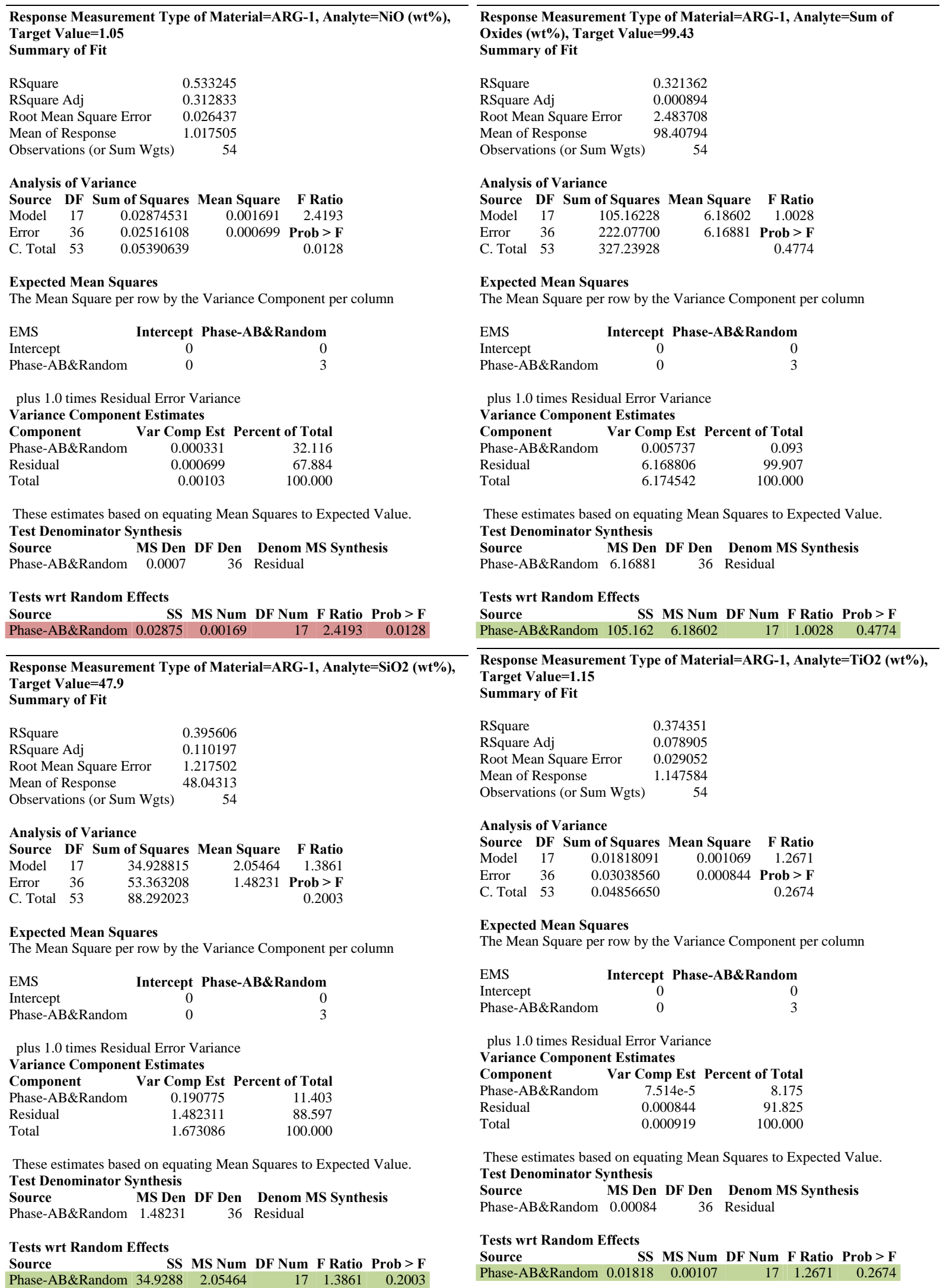




\section{Exhibit A3. Analysis of Variance to Estimate Analytical Block (AB) Effects}

\begin{tabular}{|c|c|c|c|c|}
\hline \multicolumn{5}{|c|}{$\begin{array}{l}\text { Response Measur } \\
\text { Target Value }=0.0 \\
\text { Summary of Fit }\end{array}$} \\
\hline \multicolumn{3}{|l|}{ RSquare } & \multicolumn{2}{|c|}{0.324582} \\
\hline \multicolumn{3}{|c|}{ RSquare Adj } & .005635 & \\
\hline \multicolumn{3}{|c|}{ Root Mean Square Error } & .001376 & \\
\hline \multicolumn{3}{|c|}{ Mean of Response } & 0.02441 & \\
\hline \multicolumn{3}{|c|}{ Observations (or Sum Wgts) } & 54 & \\
\hline \multicolumn{5}{|c|}{ Analysis of Variance } \\
\hline Model & 17 & 0.00003274 & $1.9262 \mathrm{e}-6$ & 1.017 \\
\hline Error & 36 & 0.00006814 & $1.8927 \mathrm{e}-6$ & Prob $=$ \\
\hline C. Total & 53 & 0.00010088 & & \\
\hline
\end{tabular}

Expected Mean Squares

The Mean Square per row by the Variance Component per column

\begin{tabular}{lcr} 
EMS & Intercept & Phase-AB\&Random \\
Intercept & 0 & 0 \\
Phase-AB\&Random & 0 & 3 \\
\multicolumn{4}{c}{ plus 1.0 times Residual Error Variance } \\
Variance Component Estimates \\
Component & Var Comp Est & Percent of Total \\
Phase-AB\&Random & $1.115 \mathrm{e}-8$ & 0.585 \\
Residual & $1.893 \mathrm{e}-6$ & 99.415 \\
Total & $1.904 \mathrm{e}-6$ & 100.000
\end{tabular}

These estimates based on equating Mean Squares to Expected Value.

Test Denominator Synthesis

Source MS Den DF Den Denom MS Synthesis

Phase-AB\&Random 1.89e-6 36 Residual

Tests wrt Random Effects

Source SS MS Num DF Num F Ratio Prob $>$ F

$\begin{array}{lrrrrr}\text { Phase-AB\&Random } & 3.27 \mathrm{e}-5 & 1.93 \mathrm{e}-6 & 17 & 1.0177 & 0.4635\end{array}$

Response Measurement Type of Material=ARG-1, Analyte=ZrO2 (wt\%),

Target Value $=0.13$

Summary of Fit

RSquare $\quad 0.384584$

RSquare Adj $\quad 0.093971$

Root Mean Square Error $\quad 0.004641$

Mean of Response $\quad 0.13182$

Observations (or Sum Wgts) 54

Analysis of Variance

Source DF Sum of Squares Mean Square F Ratio

$\begin{array}{lrrrr}\text { Model } & 17 & 0.00048462 & 0.000029 & 1.3234\end{array}$

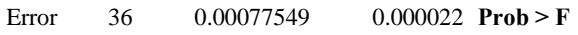

$\begin{array}{lll}\text { C. Total } 53 & 0.00126011 & 0.2337\end{array}$

Expected Mean Squares

The Mean Square per row by the Variance Component per column

$\begin{array}{lrr}\text { EMS } & \text { Intercept } & \text { Phase-AB\&Random } \\ \text { Intercept } & 0 & 0 \\ \text { Phase-AB\&Random } & 0 & 3\end{array}$

Phase-AB\&Random $\quad 0$

plus 1.0 times Residual Error Variance

Variance Component Estimates

Component Var Comp Est Percent of Total

$\begin{array}{lr}\text { Phase-AB\&Random } & \text { 2.322e-6 }\end{array}$

$\begin{array}{llr}\text { Residual } & 2.154 \mathrm{e}-5 & 90.270\end{array}$

$\begin{array}{lll}\text { Total } & 2.386 \mathrm{e}-5 & 100.000\end{array}$

These estimates based on equating Mean Squares to Expected Value.

Test Denominator Synthesis

Source MS Den DF Den Denom MS Synthesis

Phase-AB\&Random $\quad 2.15 \mathrm{e}-5 \quad 36$ Residual

Tests wrt Random Effects

Source SS MS Num DF Num F Ratio Prob $>$ F

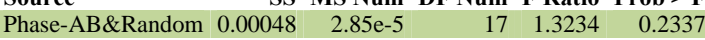




\section{Distribution:}

\begin{tabular}{|c|c|}
\hline Name: & Location: \\
\hline Sharon Marra & 773-A \\
\hline Connie Herman & 999-W \\
\hline Charles J. Coleman & 773-A \\
\hline Damon Click & 773-A \\
\hline Clint Gregory & 773-A \\
\hline Mark Barnes & 773-A \\
\hline Patricia Lee & $703-41 \mathrm{~A}$ \\
\hline Richard Walker & $703-41 \mathrm{~A}$ \\
\hline Michael Stone & 999-W \\
\hline David Peeler & 999-W \\
\hline Kevin Hera & 723-A \\
\hline Tommy Edwards & 999-W \\
\hline John Pareizs & 773-A \\
\hline Kevin Fox & 999-W \\
\hline Fabienne Johnson & 999-W \\
\hline Charles Crawford & $773-42 \mathrm{~A}$ \\
\hline David Best & 999-W \\
\hline John Occhipinti & $704-S$ \\
\hline Jonathan Bricker & $704-27 \mathrm{~S}$ \\
\hline John Iaukea & 704-30S \\
\hline Aaron Staub & $704-27 S$ \\
\hline Jeff Ray & 704-S \\
\hline Perry Bovan & $704-27 \mathrm{~S}$ \\
\hline Robert Hinds & 704-S \\
\hline Terri Fellinger & $704-26 S$ \\
\hline Ryan McNew & $704-S$ \\
\hline Michael T. Hart & $210-S$ \\
\hline Roger N. Mahannah & $704-28 S$ \\
\hline Michael T. Feller & $704-28 S$ \\
\hline Omar Cardona-Quiles & $704-24 S$ \\
\hline Amanda Shafer & $704-27 S$ \\
\hline Mason Clark & $704-27 \mathrm{~S}$ \\
\hline Helen Boyd & $704-27 \mathrm{~S}$ \\
\hline Hank Elder & $704-24 S$ \\
\hline Bill Holtzscheiter & $704-15 S$ \\
\hline Pat Vaughan & $773-41 \mathrm{~A}$ \\
\hline
\end{tabular}

